\title{
IMPLICIT-EXPLICIT RUNGE-KUTTA SCHEMES FOR HYPERBOLIC SYSTEMS AND KINETIC EQUATIONS IN THE DIFFUSION LIMIT
}

\author{
S. BOSCARINO*, L. PARESCHI ${ }^{\dagger}$, AND G.RUSSO ${ }^{\ddagger}$
}

\begin{abstract}
We consider Implicit-Explicit (IMEX) Runge-Kutta (R-K) schemes for hyperbolic systems with stiff relaxation in the so-called diffusion limit. In such regime the system relaxes towards a convection-diffusion equation. The first objective of the paper is to show that traditional partitioned IMEX R-K schemes will relax to an explicit scheme for the limit equation with no need of modification of the original system. Of course the explicit scheme obtained in the limit suffers from the classical parabolic stability restriction on the time step. The main goal of the paper is to present an approach, based on IMEX R-K schemes, that in the diffusion limit relaxes to an IMEX R-K scheme for the convection-diffusion equation, in which the diffusion is treated implicitly. This is achieved by an original reformulation of the problem, and subsequent application of IMEX R-K schemes to it. An analysis on such schemes to the reformulated problem shows that the schemes reduce to IMEX R-K schemes for the limit equation, under the same conditions derived for hyperbolic relaxation [8]. Several numerical examples including neutron transport equations confirm the theoretical analysis.
\end{abstract}

Key words. IMEX Runge-Kutta methods, hyperbolic conservation laws with sources, transport equations, diffusion equations, stiff systems.

AMS subject classification. 65C20, 65M06, 76D05, 82C40.

1. Introduction. The development of numerical methods to solve hyperbolic systems in diffusive regimes has been a very active area of research in the last years (see for example [25, 28, 31, 34).

Classical fields of applications involve diffusion in neutron transport 9, 16, 19, 26, 32, drift-diffusion limit in semiconductors [27, 30] and incompressible Navier-Stokes limits in rarefied gas dynamic [29. A strictly related field of research concerns the construction of schemes for the compressible Navier-Stokes limit (see [3] and the references therein). In such physical problems, the scaling parameter (mean free path) may differ in several orders of magnitude from the rarefied regimes to the diffusive regimes, and it is desirable to develop a class of robust numerical schemes that can work uniformly with respect to this parameter.

A prototype hyperbolic system of conservation laws with diffusive relaxation that we will use to illustrate the subsequent theory is the following, 25, 27, 34]

$$
\begin{aligned}
& u_{t}+v_{x}=0, \\
& v_{t}+\frac{1}{\varepsilon^{2}} p(u)_{x}=-\frac{1}{\varepsilon^{2}}(v-q(u)),
\end{aligned}
$$

where $p^{\prime}(u)>0$. System (1.1) is hyperbolic with two distinct real characteristics speeds $\pm \sqrt{p^{\prime}(u)} / \varepsilon$.

In the small relaxation limit, $\varepsilon \rightarrow 0$, the behavior of the solution to (1.1) is, at least formally, governed by the convection-diffusion equation

$$
\begin{aligned}
& u_{t}+q(u)_{x}=p(u)_{x x}, \\
& v=q(u)-p(u)_{x} .
\end{aligned}
$$

The so called subcharacteristic condition [15] for system (1.1) becomes

$$
\left|q^{\prime}(u)\right|^{2}<\frac{p^{\prime}(u)}{\varepsilon^{2}}
$$

and is naturally satisfied in the limit $\varepsilon \rightarrow 0$.

About the boundary conditions for system (1.1), we have to specify the domain in which we solve the problem. In a finite domain $x \in[a, b]$, one can use periodic boundary conditions, or can assign one independent condition at each boundary, since the two characteristic velocities have opposite sign.

In practice, we shall assign two conditions at each boundary, one independent and the other compatible with the equations. Furthermore, we shall choose boundary conditions which are compatible to the

\footnotetext{
* Mathematics and Computer Science Department, University of Catania, Italy (boscarino@dmi.unict.it).

$\dagger^{\dagger}$ Mathematics Department, University of Ferrara, Italy (Iorenzo.pareschi@unife.it).

${ }_{\ddagger}^{\ddagger}$ Mathematics and Computer Science Department, University of Catania, Italy (russo@dmi.unict.it).
} 
limit solution as $\varepsilon \rightarrow 0$. For example for system (1.1), if we set $v=q(u)$ at $x=a$ and $x=b$, compatibility with system (1.1) requires

$$
\varepsilon^{2} q^{\prime}(u) v_{x}=p^{\prime}(u) u_{x}, \quad x=a, b .
$$

Such condition becomes $u_{x}=0$ in the limit $\varepsilon \rightarrow 0$. In the sections on the numerical tests we shall specify the boundary conditions we use in each test.

In general, numerical approaches that work for hyperbolic system with stiff relaxation terms do not apply directly in the diffusive scaling since in these systems we have the presence of multiple time-scales.

In fact, together with the stiff relaxation term we have a stiff convection term that contributes to the asymptotic diffusive behavior. Then special care must be taken to ensure that the schemes possess the correct zero-relaxation limit, in the sense that the numerical scheme applied to system (1.1) should be a consistent and stable scheme for the limit system (1.2) as the parameter $\varepsilon$ approaches zero independently of the discretization parameters. A notion usually referred to as asymptotic preservation. For a nice survey on asymptotic preserving scheme for various kinds of systems see, for example, the review paper by Shi Jin ([23]). Furthermore, a different approach to the derivation of asymptotic preserving schemes is described in the review by Pierre Degond [17. In the case of Boltzmann kinetic equations we also refer to the recent review by two of the authors 37 .

IMEX Runge-Kutta (R-K) schemes 1, 5, 6, 11, 36 represent a powerful tool for the time discretization of such stiff systems. Unfortunately, since the characteristic speed of the hyperbolic part is of order $1 / \varepsilon$, standard IMEX R-K schemes developed for hyperbolic systems with stiff relaxation [36, 8, become useless in such parabolic scaling, because the CFL condition would require $\Delta t=\mathcal{O}(\varepsilon \Delta x)$. Of course, in the diffusive regime where $\varepsilon<\Delta x$, this is very restrictive since for an explicit method a parabolic condition $\Delta t=\mathcal{O}\left(\Delta x^{2}\right)$ should suffice.

Most previous works on asymptotic preserving schemes for hyperbolic systems and kinetic equations with diffusive relaxation focus on schemes which in the limit of infinite stiffness become consistent explicit schemes for the diffusive limit equation [25, 28, 31, 32, 34. Such schemes have been derived by splitting the stiff hyperbolic part into an explicit (non-stiff) term, and an implicit (stiff) term. Here we show that by applying partitioned IMEX R-K schemes, in which the stiffness is associated with the variable and not with the operator, one obtains IMEX R-K schemes that naturally relax to the explicit scheme applied to the limit convection-diffusion equation. All these explicit schemes clearly suffer from the usual stability restriction $\Delta t=\mathcal{O}\left(\Delta x^{2}\right)$.

In this paper we present a general methodology to overcome such stability restriction which applies to a broad class of problems. The idea is to reformulate problem (1.1) by properly combining the limiting diffusion flux with the convective flux. This allows to construct a class of IMEX R-K schemes that work with high order accuracy in time and that, in the diffusion limit (i.e. when $\varepsilon \rightarrow 0$ ), originate an IMEX method for the limiting convection-diffusion equation (1.2). Other reformulations whose goal is to obtain asymptotic-preserving methods have been proposed in [25, 24]. Schemes that avoid such time step restriction and provide fully implicit solvers in the case of transport equations have been analyzed in [9].

Our new approach allows a hyperbolic CFL condition $\Delta t=\mathcal{O}(\Delta x)$ independent of $\varepsilon$ when applied to (1.1) in all regimes. The aim of this paper is to derive and analyze different types of IMEX R-K schemes when applied to the reformulated problem in the stiff regime $(\varepsilon \rightarrow 0)$.

The rest of the paper is organized as follows. The next section is devoted to partitioned IMEX R-K schemes. It is shown that they relax to the explicit scheme applied to the convection diffusion limit. In Section 3 the new approach is introduced and analyzed. In particular, following [8, we prove that under suitable assumptions the IMEX R-K schemes are consistent with the diffusion limit. The analysis is based on a power series expansion in $\varepsilon$ of the exact and numerical solution. It is shown that, at lowest order in $\varepsilon$, the model system is a set of differential-algebraic equations of index 1, i.e. it can be transformed into a set of ordinary differential equations by one time differentiation. Compatibility between exact and numerical solution at different orders in $\varepsilon$ introduces additional order conditions on the coefficients of the IMEX schemes. To the lowest order such conditions are referred as index 1 order conditions.

After a short section on space discretization obtained by conservative finite difference schemes, in Section 5 we report several numerical examples and tests. In Section 6 we consider one-dimensional 
neutron transport equation and present several numerical results and comparison with schemes available in the literature. Additional technical material is given in the separate Appendices.

2. Partitioned IMEX R-K schemes. The first observation is that in system (1.1) the stiffness is naturally associated to the variable $v$ rather then to some operator. The system has the structure of a singular perturbation problem 21, and it can be treated by a partitioned R-K scheme in which the first equation is treated explicitly and the second implicitly

$$
\begin{array}{ll}
u_{t}=-v_{x}, & \text { (Explicit) } \\
\varepsilon^{2} v_{t}=-\left(p(u)_{x}+v-q(u)\right) . & \text { (Implicit) }
\end{array}
$$

This approach has been used, for example, in [28, 35].

By using a method of lines approach (MOL), we discretize system (2.1) in space by a uniform mesh $\left\{x_{i}\right\}_{i=1}^{N}$ and $U_{i}(t) \approx u\left(x_{i}, t\right), V_{i}(t) \approx v\left(x_{i}, t\right)$. We obtain a large system of ODE's

$$
\begin{aligned}
& U_{t}=\mathcal{F}(V), \\
& \varepsilon^{2} V_{t}=G(U)-V,
\end{aligned}
$$

with $U(t)=\left(U_{1}(t), U_{2}(t), \ldots, U_{N}(t)\right)^{T} \in \mathbb{R}^{N}$ and $V(t)=\left(V_{1}(t), V_{2}(t), \ldots, V_{N}(t)\right)^{T} \in \mathbb{R}^{N}$, where $\mathcal{F}(V)=$ $-D V$ and $G(U)=Q(U)-D p(U)$. Here $D V$ and $D p(U)$ (with a slight abuse of notation) denote the discretization of the convective terms $v_{x}, p(u)_{x}$, while $Q(U)$ represents the discretization of the term $q(u)$.

As we shall see, if the implicit scheme is $L$-stable [21], in the limit $\varepsilon \rightarrow 0$ the IMEX R-K scheme will relaxe to the explicit scheme applied to the limit equation

$$
U_{t}=: \hat{\mathcal{F}}(U),
$$

where $\hat{\mathcal{F}}(U)=\mathcal{F}(G(U))$.

For example, implicit-explicit Euler scheme applied to system (2.2) gives

$$
\begin{aligned}
& U_{n+1}=U_{n}+\Delta t \mathcal{F}\left(V_{n}\right) \\
& V_{n+1}=\frac{\varepsilon^{2} V_{n}+\Delta t G\left(U_{n+1}\right)}{\varepsilon^{2}+\Delta t},
\end{aligned}
$$

where we have discretized the interval of integration by a time mesh $\left\{t_{n}\right\}_{n=1}^{\mathcal{N}}$ and $U_{n} \approx U\left(t_{n}\right)$. As $\varepsilon \rightarrow 0$, $V_{n+1}=G\left(U_{n+1}\right)$ and therefore $U_{n+1}=U_{n}+\Delta t \hat{\mathcal{F}}\left(U_{n}\right)$.

In the case $p(u)=u$ and $q(u)=0$, the method would relax to explicit Euler scheme applied to the diffusion equation, thus suffering the usual parabolic CFL stability condition $\Delta t \leqslant \Delta x^{2} / 2$. This approach will be denoted as partitioned approach.

2.1. Classification of IMEX R-K schemes. IMEX R-K schemes have been widely used in the literature to treat problems that contain both stiff and non stiff terms [1, 11, 36]. The stiff terms are treated implicitly, while the non stiff terms are treated explicitly, thus lowering the computational complexity of the scheme.

Usually such a scheme is characterized by the $s \times s$ matrices $\tilde{A}=\left(\tilde{a}_{i j}\right), A=\left(a_{i j}\right)$ and the vectors $\tilde{b}$, $b \in \mathbb{R}^{s}$, and can be represented by a double tableau in the usual Butcher notation

$$
\begin{array}{l|l}
\tilde{c} & \tilde{A} \\
\hline & b^{T}
\end{array}, \begin{array}{l|l}
c & A \\
\hline & b^{T}
\end{array} .
$$

The coefficients $\tilde{c}$ and $c$ are used if the right hand side depends explicitly on time. We assume that they satisfy the usual relation

$$
\tilde{c}_{i}=\sum_{j=1}^{i-1} \tilde{a}_{i j}, \quad c_{i}=\sum_{j=1}^{i} a_{i j}
$$


Matrix $\tilde{A}$ is lower triangular with zero diagonal, while matrix $A$ is lower triangular, i.e. the implicit scheme is a diagonally implicit Runge-Kutta (DIRK). This choice guarantees that the term $\mathcal{F}(V)$ in (2.2) is always explicitly evaluated.

IMEX R-K schemes presented in the literature can be classified in two main different types characterized by the structure of the matrix $A=\left(a_{i j}\right)_{i, j=1}^{s}$ of the implicit scheme.

Definition 2.1. We call an IMEX $R$-K method of type $A$ (see [36]) if the matrix $A \in \mathbb{R}^{s \times s}$ is invertible.

Definition 2.2. We call an IMEX $R$-K method of type $C K$ (see [11]) if the matrix $A \in R^{s} \times s$ can be written as

$$
A=\left(\begin{array}{ll}
0 & 0 \\
a & \hat{A}
\end{array}\right)
$$

with $a \in \mathbb{R}^{(s-1)}$ and the submatrix $\hat{A} \in \mathbb{R}^{(s-1) \times(s-1)}$ invertible. In the special case $a=0$ the scheme is said to be of type ARS (see [1]).

We note that schemes CK are very attractive because they allow some simplifying assumptions, that make order conditions easier to treat, therefore permitting the construction of higher order IMEX R-K schemes. On the other hand, schemes of type A are more amenable to a theoretical analysis, since the matrix $A$ of the implicit scheme is invertible. This is why we start our analysis with the latter schemes.

2.2. Analysis of IMEX schemes for the partitioned approach. Now as an example we perform the analysis of type A scheme when applied to system (2.1). A similar analysis is possible also for CK schemes. We will restrict our analysis to the limit case $\varepsilon \rightarrow 0$.

Applying an IMEX R-K scheme to system (2.2) we obtain

$$
\begin{aligned}
U_{n+1} & =U_{n}+\Delta t \sum_{k=1}^{s} \tilde{b}_{k} \mathcal{F}\left(\mathcal{V}_{k}\right), \\
\varepsilon^{2} V_{n+1} & =\varepsilon^{2} V_{n}+\Delta t \sum_{k=1}^{s} b_{k}\left(G\left(\mathcal{U}_{k}\right)-\mathcal{V}_{k}\right),
\end{aligned}
$$

for the numerical solution and

$$
\begin{gathered}
\mathcal{U}_{k}=U_{n}+\Delta t \sum_{j=1}^{k-1} \tilde{a}_{k j} \mathcal{F}\left(\mathcal{V}_{j}\right) \\
\varepsilon^{2} \mathcal{V}_{k}=\varepsilon^{2} V_{n}+\Delta t \sum_{j=1}^{k} a_{k j}\left(g\left(\mathcal{U}_{j}\right)-\mathcal{V}_{j}\right),
\end{gathered}
$$

for the internal stages.

By Definition 2.1 and $A$ invertible we obtain from the second equation in (2.5)

$$
\Delta t\left(G\left(\mathcal{U}_{k}\right)-\mathcal{V}_{k}\right)=\varepsilon^{2} \sum_{j=1}^{k} \omega_{k j}\left(\mathcal{V}_{j}-V_{n}\right) .
$$

From now on, $\omega_{k j}$ are the elements of the inverse matrix $A^{-1}$. Now inserting (2.7) into the numerical solution $V_{n+1}$ and setting $\varepsilon=0$, we get

$$
V_{n+1}=\mathcal{R}(\infty) V_{n}+\Delta t \sum_{k=1}^{s} b_{k} \omega_{k j} \mathcal{V}_{j}
$$

Here we denoted by

$$
\mathcal{R}(\infty)=1-b^{T} A^{-1} \mathbf{1}=\lim _{z \rightarrow \infty} \mathcal{R}(z),
$$


where $\mathcal{R}(z)$ is the stability function of the implicit scheme defined by (see [21], Sect. IV.3)

$$
\mathcal{R}(z)=1+z b^{T}(I-z A)^{-1} \mathbf{1},
$$

with $b^{T}=\left(b_{1}, \ldots, b_{s}\right)$ and $\mathbf{1}=(1, \ldots, 1)^{T}$.

By (2.7) we get $\mathcal{V}_{k}=G\left(\mathcal{U}_{k}\right)$ when $\varepsilon=0$. This yields that $\hat{\mathcal{F}}\left(\mathcal{U}_{k}\right)=\mathcal{F}\left(G\left(\mathcal{U}_{k}\right)\right)$, and we obtain

$$
U_{n+1}=U_{n}+\Delta t \sum_{k=1}^{s} \tilde{b}_{k} \hat{\mathcal{F}}\left(\mathcal{U}_{k}\right)
$$

with

$$
\mathcal{U}_{k}=U_{n}+\Delta t \sum_{j=1}^{k-1} \tilde{a}_{k j} \hat{\mathcal{F}}\left(\mathcal{U}_{j}\right)
$$

internal stages and $k=1, \ldots, s$. This represents the explicit scheme of the starting IMEX R-K one of type A applied to the limit equation (2.3) obtained by (2.2) when $\varepsilon \rightarrow 0$. As particular case, if $p(u)=u$ and $q(u)=0$, this is the explicit scheme applied to the limit diffusion equation under the usual parabolic stability restriction $\left(\Delta t \leqslant \Delta x^{2} / 2\right)$.

3. Overcoming parabolic stiffness. In order to overcome such stability restriction, we reformulate system (1.1) as the equivalent system

$$
\begin{aligned}
& u_{t}=-\left(v+\mu p(u)_{x}\right)_{x}+\mu p(u)_{x x}, \\
& \varepsilon^{2} v_{t}=-p(u)_{x}-v+q(u),
\end{aligned}
$$

where the term $\mu p(u)_{x x}$ has been added and subtracted to the first equation in (1.1). Here $\mu=\mu(\varepsilon) \in[0,1]$ is a free parameter such that $\mu(0)=1$. The idea is that, since the quantity $v+p(u)_{x}$ is close to $q(u)$ as $\varepsilon \rightarrow 0$, the first term on the right hand side can be treated explicitly in the first equation, while the term $p(u)_{x x}$ will be treated implicitly. This can be done naturally by using an Implicit-Explicit approach, as we will explain later. Let us point out that the choice $\mu \equiv 1$, as shown in Appendix 8.1 for a first order implicit-explicit scheme, guarantees the largest stability region of the method.

Next we will study the behavior of the different IMEX R-K schemes when applied to system (3.1) in the diffusion limit. In particular we will show that such schemes reduce to the same IMEX R-K schemes for the limit equation and no parabolic stability restriction on the time step appears in the diffusive limit.

3.1. The new approach. System (3.1) can be written in the form

$$
\begin{aligned}
& u^{\prime}=f_{1}(u, v)+f_{2}(u), \\
& \varepsilon^{2} v^{\prime}=g(u, v)
\end{aligned}
$$

where the primes denote the time derivatives and

$$
\begin{gathered}
f_{1}(u, v)=-\left(v+\mu p(u)_{x}\right)_{x}, \quad f_{2}(u)=\mu p(u)_{x x}, \\
g(u, v)=-p(u)_{x}-v+q(u) .
\end{gathered}
$$

Notice that, throughout this paper, $g(u, v)$ (and therefore $g_{v}(u, v)$ ), depends only algebraically on $v$, while it may contain differential operators acting on $u$.

Now we apply an IMEX-RK scheme to system (3.2) where $\left(f_{1}(u, v), 0\right)^{T}$ is evaluated explicitly and $\left(f_{2}(u), g(u, v)\right)^{T}$ implicitly. Note that if $f_{2}(u)$ is evaluated explicitly then by cancelation the IMEX-RK scheme will reduce to the typology of asymptotic preserving methods studied in [7, 35.

In the limit $\varepsilon \rightarrow 0$ from (3.2) we obtain a differential algebraic system (DAE)

$$
\begin{aligned}
& u^{\prime}=f_{1}(u, v)+f_{2}(u), \\
& 0=g(u, v) .
\end{aligned}
$$


In order to guarantee the solvability of system (3.3) we assume that the Jacobian matrix $g_{v}(u, v)$ is invertible, and then the DAE is said to be of index one 11. Note that if $g_{v}$ has a bounded inverse in a neighborhood of the exact solution, we can use the inverse function theorem in order to write

$$
v(t)=G(u(t))
$$

for some $G(u)$ which inserted into $u^{\prime}=f_{1}(u, v)+f_{2}(u)$ gives $u^{\prime}=f_{1}(u, G(u))+f_{2}(u)$. From now on we always assume that this is the case. Then, as $\varepsilon \rightarrow 0$ system (3.2) reduces to

$$
u^{\prime}=\hat{f}_{1}(u)+f_{2}(u)
$$

where $\hat{f}_{1}(u)=f_{1}(u, G(u))$ and $v=G(u)$. This approach will be denoted BPR approach.

First order implicit-explicit Euler scheme that uses this approach is reported in Appendix 8.1, where a stability analysis is performed. In particular it is shown that as $\varepsilon \rightarrow 0$, the parabolic restriction on time step disappears.

In the sequel we restrict our analysis to the limit case $\varepsilon \rightarrow 0$ where the main goal is to capture the diffusive limit.

3.2. Analysis of TYPE A IMEX schemes. Applying an IMEX R-K scheme of type A to system (3.2) we obtain

$$
\begin{aligned}
u_{n+1} & =u_{n}+\Delta t \sum_{k=1}^{s} \tilde{b}_{k} f_{1}\left(U_{k}, V_{k}\right)+\Delta t \sum_{k=1}^{s} b_{k} f_{2}\left(U_{k}\right) \\
\varepsilon^{2} v_{n+1} & =\varepsilon^{2} v_{n}+\Delta t \sum_{k=1}^{s} b_{k} g\left(U_{k}, V_{k}\right),
\end{aligned}
$$

for the numerical solution and

$$
\begin{aligned}
U_{k} & =u_{n}+\Delta t \sum_{j=1}^{k-1} \tilde{a}_{k j} f_{1}\left(U_{j}, V_{j}\right)+\Delta t \sum_{j=1}^{k} a_{k j} f_{2}\left(U_{j}\right) \\
\varepsilon^{2} V_{k} & =\varepsilon^{2} v_{n}+\Delta t \sum_{j=1}^{k} a_{k j} g\left(U_{j}, V_{j}\right),
\end{aligned}
$$

for the internal stages (notice a slight changes of notation with respect to Section 2).

Starting from (3.5) and (3.6), by Definition (2.1) and $A$ invertible, we obtain from the second equation in (3.6)

$$
\Delta t g\left(U_{k}, V_{k}\right)=\varepsilon^{2} \sum_{j=1}^{k} \omega_{k j}\left(V_{j}-v_{n}\right),
$$

Inserting this into the numerical solution $v_{n+1}$ we make $v_{n+1}$ independent of $\varepsilon^{2}$ and setting $\varepsilon=0$, we get

$$
\begin{aligned}
& u_{n+1}=u_{n}+\Delta t \sum_{k=1}^{s} \tilde{b}_{k} \hat{f}_{1}\left(U_{k}\right)+\Delta t \sum_{k=1}^{s} b_{k} f_{2}\left(U_{k}\right) \\
& v_{n+1}=\mathcal{R}(\infty) v_{n}+\Delta t \sum_{k=1}^{s} b_{k} \omega_{k j} V_{j},
\end{aligned}
$$

\footnotetext{
${ }^{1}$ The index of a DAE is the number of times one has to differentiate the function $g$ to obtain a system of ODE's. For example, differentiating the function $g$, one obtains $g_{u}(u, v) u^{\prime}+g_{v}(u, v) v^{\prime}=0$. If $g_{v}$ is invertible, system (3.3) can be written as $u^{\prime}=f(u, v), v^{\prime}=-g_{v}^{\prime} g_{u} f$.
} 
with $\hat{f}_{1}\left(U_{k}\right)=f_{1}\left(U_{k}, G\left(U_{k}\right)\right)$, and stage values

$$
\begin{aligned}
U_{k} & =u_{n}+\Delta t \sum_{j=1}^{k-1} \tilde{a}_{k j} \hat{f}_{1}\left(U_{j}\right)+\Delta t \sum_{j=1}^{k} a_{k j} f_{2}\left(U_{j}\right) \\
0 & =g\left(U_{k}, V_{k}\right) .
\end{aligned}
$$

for $k=1, \ldots, s$. The latter equality implies $V_{k}=G\left(U_{k}\right), k=1, \ldots, s$.

Note that if we require that the implicit part of the scheme is stiffly accurate, i.e. if

$$
b^{T} A^{-1}=e_{s}^{T},
$$

where $e_{s}=(0, \ldots, 0,1)^{T}$, then by (2.9)

$$
\mathcal{R}(\infty)=1-b^{T} A^{-1} \mathbf{1}=1-e_{s}^{T} \mathbf{1}=1-1=0 .
$$

This implies that if the implicit scheme is $A$-stable and stiffly accurate it is also $L$-stable and $v_{n+1}=$ $V_{s}=G\left(U_{s}\right)$.

It is interesting to note that, if we consider system (3.1) with $q(u)=0$, when $\varepsilon=0$ we get a purely diffusive system which means that the term $f_{1}(u, v)$ in (3.2) disappears. Therefore, by BPR approach, the IMEX R-K scheme of type A in the limit $\varepsilon \rightarrow 0$ becomes a stiffly accurate DIRK scheme and hence no stability restriction on the time step is required in the diffusive limit, i.e. we got an unconditionally stable method. Another advantage of this new approach is the following. Usually the numerical solution $\left(u_{n+1}, v_{n+1}\right)$ in (3.7) in the case $\varepsilon=0$ will not lie on the manifold $g(u, v)=0$ since $g\left(u_{n+1}, v_{n+1}\right)$ is not necessarily zero. But this approach guarantees that in the limit $\varepsilon \rightarrow 0$ we obtain a stiffly accurate implicit scheme and hence $u_{n+1}=U_{s}$, implying $g\left(u_{n+1}, v_{n+1}\right)=0$.

In the general case of systems for which $q(u) \neq 0$, it is $f_{1}(u, v) \neq 0$ and, by using the BPR approach, in the limit case $\varepsilon \rightarrow 0$ we obtain an IMEX R-K scheme with a non vanishing explicit term in which the diffusion term $f_{2}(u)$ is treated implicitly and a classical CFL hyperbolic condition for the time step is required. In general $g\left(u_{n+1}, v_{n+1}\right) \neq 0$ even if all stage values lie on the manifold, (see the second equation in (3.8)). However, if the explicit scheme has the property that $u_{n+1}=U_{s}$, and the implicit scheme is stiffly accurate, then, in the limit as $\varepsilon \rightarrow 0$ the numerical solutions are projected on the manifold $g\left(u_{n+1}, v_{n+1}\right)=0$, because $g\left(u_{n+1}, v_{n+1}\right)=g\left(U_{s}, V_{s}\right)=0$.

From the above discussion it is clear that the property $u_{n+1}=U_{s}$ is crucial if we want that the numerical solution is projected to the limit manifold as $\varepsilon \rightarrow 0$. We emphasize that there is a class of $s$-stage explicit R-K methods for which $u_{n+1}=U_{s}$; such methods are called First Same As Last (FSAL), and their coefficients satisfy $a_{s, i}=b_{i}$ for $i=1, \ldots, s-1$ and $b_{s}=0$. They have the advantage of requiring $s-1$ function evaluations for each step (see 22] for details). FSAL methods are often used in the contest of embedded methods, such as the popular Dormond-Prince method (DOPRI) [18, on which MATLAB routine ode 45 is based on.

From the arguments above, in order to capture the limit as $\varepsilon \rightarrow 0$, it is important that the implicit part on an IMEX R-K is stiffly accurate and the explicit part is FSAL. This motivates the following

DeFINITION 3.1. We say that a IMEX $R$-K scheme is globally stiffly accurate if $b^{T}=e_{s}^{T} A$ and $\tilde{b}^{T}=e_{s}^{T} \tilde{A}$, with $e_{s}=(0, \ldots, 0,1)^{T}$, and $c_{s}=\tilde{c}_{s}=1$, i.e. the numerical solution is identical to the last internal stage value of the scheme.

From (3.5) and (3.6) we observe that if an IMEX R-K is globally stiffly accurate, then $u_{n+1}=U_{s}$, $v_{n+1}=V_{s}$, and therefore $\lim _{\varepsilon \rightarrow 0} g\left(u_{n+1}, v_{n+1}\right)=0$.

General remarks for type $A$.

- It is worth mentioning some important aspects about type A schemes. First of all, in $[5]$ Boscarino emphasized that an important ingredient for the IMEX R-K schemes of type A is $b_{i}=\tilde{b}_{i}$ for all $i$. Such a choice provides a significant benefit for the differential component $u$, i.e., an order reduction does not appear for this component. On the other hand, conditions

$$
e_{s}^{T} \tilde{A}=\tilde{b}^{T}, \quad e_{s}^{T} A=b^{T}
$$

imply $a_{s s}=b_{s} \neq \tilde{b}_{s}=\tilde{a}_{s s}=0$ which means that for a stiffly accurate IMEX R-K scheme it is $b \neq \tilde{b}$, and therefore we expect to observe order reduction for the differential variable. 
- It is impossible to construct a second order stiffly accurate IMEX R-K scheme of type A with $s=3$ internal stages. The proof is given in Appendix 8.2 In practice, in order to satisfy all these order conditions we have to increase the number of the internal stages. In view of such difficulties, for type A schemes, we shall consider second order IMEX R-K schemes with $s=3$ and $\tilde{b}=b$ in order to avoid the order reduction, giving up to the FSAL property of the explicit scheme (and with that the global stiff accuracy of the IMEX scheme). An example is the scheme $\operatorname{SSP}(3,3,2)$ in Appendix 8.3. In this case, if $q(u) \neq 0$, it is $g\left(u_{n+1}, v_{n+1}\right) \neq 0$ as $\varepsilon \rightarrow 0$.

- Formulation (3.2) in the limit case $\varepsilon \rightarrow 0$ yields the index-1 DAE. Then using the same technique adopted in [6], we can derive additional order conditions, called algebraic conditions, that guarantee the correct behavior of the numerical solution in the limit $\varepsilon \rightarrow 0$ and maintain the accuracy in time of the scheme. If the implicit scheme is stiffly accurate, such conditions becomes, to various order of accuracy,

$$
\begin{aligned}
& \tilde{c}_{s}=1, \quad(\text { second order }) \\
& e_{s}^{T} \tilde{A} \tilde{c}=1 / 2, \quad(\text { third order })
\end{aligned}
$$

where $e_{s}=(0, \ldots, 0,1)^{T}$. If the IMEX schemes is globally stiffly accurate, then (3.9) are automatically satisfied, since $e_{s}^{T} \tilde{A}=\tilde{b}^{T}$.

- Finally we observe that, in order to construct an order $p \geq 3$ IMEX R-K of type A and to maintain accuracy we have to increase the number of the classical order conditions too. Usually several simplifying assumptions (see [6, 8, 21 for details) could help to reduce the number of such conditions, but, higher orders type A schemes are more complicated to construct than CK or ARS schemes because of additional order conditions (see 8 ) due to the fact that $c \neq \tilde{c}$.

3.3. Analysis of TYPE CK schemes. Similar considerations about BPR approach, explained for the IMEX R-K scheme of type A in the limit case $\varepsilon \rightarrow 0$, can be reproposed here for the type CK when applied to the system (3.3), with slightly modifications. Of course, if we consider the general system (3.1) we obtain again an IMEX R-K scheme of type CK in the diffusion limit, i.e. $\varepsilon \rightarrow 0$, where the diffusion term $f_{2}(u)$ is treated implicitly and a CFL hyperbolic condition for the time step is required.

Indeed, we consider an IMEX R-K schemes of type CK where, by Definition 2.2 we assume that the submatrix $\hat{A}$ is invertible and $a_{11}=0$. The Butcher tableaux of a CK scheme takes the form

$$
\begin{array}{c|cc}
0 & 0 & 0 \\
\hat{c} & a & \hat{A} \\
\hline & b_{1} & b^{T}
\end{array}
$$

with $a=\left(a_{21}, \ldots, a_{s, 1}\right)^{T}$ and $\hat{b}^{T}=\left(b_{2}, \ldots, b_{s}\right)$. In order to simplify the analysis we consider that the implicit part of the scheme is stiffly accurate. Under this circumstance it is easy to prove that

$$
b_{1}+\hat{b}^{T} \alpha=0
$$

where $\alpha \equiv-\hat{A}^{-1} a$ (see $[5$ for details).

Then, considering a scheme of the type CK, the second equation in (3.6) becomes

$$
\varepsilon^{2} V_{k}=\varepsilon^{2} v_{n}+\Delta t a_{k 1} g\left(u_{n}, v_{n}\right)+\Delta t \sum_{j=2}^{k} a_{k j} g\left(U_{j}, V_{j}\right) .
$$

with $k=2, \ldots, s$.

Now multiplying by $\hat{\omega}_{k j}$, where $\hat{\omega}_{k j}$ are the elements of the inverse of $\hat{A}$, and summing on $k$, we obtain

$$
\Delta t g\left(U_{k}, V_{k}\right)=\varepsilon^{2} \sum_{j=2}^{s} \hat{\omega}_{k j}\left(V_{j}-v_{n}\right)+\Delta t \alpha_{k} g\left(u_{n}, v_{n}\right), \text { for } k=2, \ldots, s
$$


where

$$
\sum_{l=2}^{s} \hat{\omega}_{k l} a_{l j}=\delta_{k j}, \quad-\sum_{l=2}^{s} \hat{\omega}_{k j} a_{j 1}=\alpha_{k} .
$$

Inserting the expression $\Delta t g\left(U_{k}, V_{k}\right)$ into the second equation in (3.5) we obtain

$$
\varepsilon^{2} v_{n+1}=\varepsilon^{2} \mathcal{R}(\infty) v_{n}+\varepsilon^{2} \Delta t \sum_{k=2}^{s} b_{k} \omega_{k j} V_{j}+\Delta t\left(b_{1}+\sum_{k=2}^{s} b_{k} \alpha_{k}\right) g\left(u_{n}, v_{n}\right)
$$

Then by (3.10) the last term in the second equation in (3.12) drops and in the limit case for $\varepsilon=0$ we can write

$$
v_{n+1}=\mathcal{R}(\infty) v_{n}+\Delta t \sum_{k=2}^{s} b_{k} \omega_{k j} V_{j}
$$

with

$$
g\left(U_{k}, V_{k}\right)=\alpha_{k} g\left(u_{n}, v_{n}\right), \text { for } k=2, \ldots, s .
$$

Note that, for IMEX R-K schemes of type CK, the stability function $\mathcal{R}(z)$ of the implicit part of the scheme takes the form

$$
\begin{aligned}
\mathcal{R}(z) & =1+z\left(b_{1}+\hat{b}^{T}(I-z \hat{A})^{-1}\left(\mathbf{1}_{s-1}+z a\right)\right) \\
& =\left(b_{1}-\hat{b}^{T} \hat{A}^{-1} a\right) z+\left(1-\hat{b}^{T} \hat{A}^{-1} \mathbf{1}_{s-1}+\hat{b}^{T} \hat{A}^{-2} a\right)+\mathcal{O}\left(\frac{1}{z}\right) .
\end{aligned}
$$

We obtained this result, by applying one step of the implicit part of the scheme to the test problem $y^{\prime}=\lambda y, y\left(t_{0}\right)=1$, with $\lambda \in \mathbb{C}$ and $\mathbf{1}_{s}=(1, \ldots, 1)^{T} \in \mathbb{R}^{s}$.

Thus, the only stiffly accurate condition, i.e. $\hat{e}_{s-1}^{T} \hat{A}=\hat{b}^{T}$ is not enough to guarantee that $\lim _{z \rightarrow \infty} \mathcal{R}(z)=$ 0 and then an additional condition is required for the implicit part of the scheme, (for details see [8]). This is expressed by the following

Proposition 3.2. If

$$
-\hat{e}_{s-1}^{T} \hat{A}^{-1} a=\sum_{j \geq 2} \hat{\omega}_{s j} a_{j 1}=0
$$

then $\mathcal{R}(\infty)=0$, where $\hat{e}_{s-1}=(0, \ldots, 0,1)^{T} \in \mathbb{R}^{s-1}$.

Proof. In fact, assuming $\hat{A}$ invertible, we get $\hat{b}^{T} \hat{A}^{-1}=\hat{e}_{s-1}^{T}$ and when $z \rightarrow \infty$, from (3.14) we obtain $\mathcal{R}(\infty)=\hat{b}^{T} \hat{A}^{-2} a=-\hat{e}_{s-1}^{T} \hat{A}^{-1} a$, which is zeros if (3.15) is satisfied.

Note that the previous Lemma implies that $\alpha_{s}=-\hat{e}_{s-1}^{T} \hat{A}^{-1} a=0$ and by (3.13) with $k=s$ we obtain $g\left(U_{s}, V_{s}\right)=0$, then the last stage values lie on the manifold $g(u, v)=0$ as $\varepsilon \rightarrow 0$. Now we observe that if the IMEX R-K scheme of type CK is globally stiffly accurate, we obtain from (3.12) and (3.7) $u_{n+1}=U_{s}$ and $v_{n+1}=V_{s}$ and therefore $g\left(u_{n+1}, v_{n+1}\right)=0$ with $v_{n+1}=G\left(u_{n+1}\right)$.

Since an IMEX R-K schemes of type ARS is a particular case of the type CK where the vector $a=0$, then the same results hold true.

General remarks for type $C K$.

- IMEX CK schemes [11 are attractive because of their good properties. The implicit part of this scheme is singly diagonally implicit Runge-Kutta (SDIRK) with $a_{i i}=\gamma>0$ for $i=2, \ldots, s$ and differs from the classical SDIRK one because $a_{11}=0$. In [11] such implicit schemes are called explicit singly diagonally implicit (ESDIRK). A consequence to set $a_{11}=0$ is the possibility to guarantee stage-order $q$ higher than the in the case of SDIRK, for which $q=1$. Moreover here we consider schemes that are stiffly accurate according to Definition 3.1 Such schemes will project the solution on the manyfold in the limit of infinite stiffness. For these schemes $b \neq \tilde{b}$, so one of the so-called simplifying conditions cannot be applied [8]. Here we require that $c_{i}=\tilde{c}_{i}$ for all $i=2, \ldots, s$; this choice will reduce the number of coupled order conditions. 
4. IMEX-Finite Difference schemes. When constructing numerical schemes, one has also to take a great care in order to avoid spurious numerical oscillations arising near discontinuities of the solution. This is avoided by a suitable choice of space discretization. To this aim it is necessary to use non-oscillatory interpolating algorithms, in order to prevent the onset of spurious oscillations (like ENO and WENO methods), see [40. Moreover the choice of the space discretization may be relevant for a correct treatment of the boundary conditions.

In this section we emphasize some requirements about the space discretization of the system (3.1). We remark that the dissipative nature of upwind schemes [34, 35] depends essentially on the fact that the characteristic speeds of the hyperbolic part are proportional to $1 / \varepsilon$. On the other hand central differences schemes avoid excessive dissipation but when $\varepsilon$ is not small or when the limiting equations contain advection terms may lead to unstable discretizations. In order to overcome these well-known facts and to have the correct asymptotic behavior we fix some general requirements for the space discretization.

1. Correct diffusion limit. Let us consider system (3.1) with $q(u)=0$. In the limit case $\varepsilon \rightarrow 0$ the therm $v+\partial_{x} p(u) \rightarrow 0$ from the second equation. If we want that $v+\mu(\varepsilon) \partial_{x} p(u) \rightarrow 0$ also in the first equation, we need to use the same space discretization for the term $\partial_{x} p(u)$ and require that $\mu(0)=1$.

2. Compact stencil. Among the advantages of our approach there is the possibility to have a scheme with a compact stencil in the diffusion limit $\varepsilon \rightarrow 0$. This property is satisfied if point 1 ) is satisfied and we use a suitable discretization for the second order derivative that characterize the diffusion limit.

3. Shock capturing. The schemes when $q(u) \neq 0$ should be based on shock capturing high order fluxes for the convection part. This is necessary not only for large values of $\varepsilon$ but also when we consider convection-diffusion type limit equations with small diffusion. The high order fluxes are then necessary for all space derivatives except for the second order term $\mu(\varepsilon) \partial_{x x} p(u)$ on the right-hand side.

4. Avoid solving nonlinear algebraic equations. In order to achieve this the implicit space derivative $\partial_{x} p(u)$ in the second equation must be evaluated using only nodal values of $u$ which can be obtained from the solution of the first equation.

The above properties are satisfied for example using high accuracy in space obtained by finite difference discretization with Weighted-Essentially Non Oscillatory (WENO) reconstruction, 40.

System (3.1) may be written in the form

$$
\begin{aligned}
u_{t}+\left(v+\mu p(u)_{x}\right)_{x} & =\mu p(u)_{x x}, \\
v_{t} & =\frac{1}{\varepsilon^{2}}\left(q(u)-\left(v+p(u)_{x}\right)\right) .
\end{aligned}
$$

with $\mu=\mu(\varepsilon)$ introduced in Section 2. The terms on the right-hand side will be treated implicitly. For large value of $\varepsilon$ the explicit flux is just $(v, 0)^{T}$, while for small values of $\varepsilon$ it is $\left(v+p(u)_{x}, 0\right)^{T}$. Here we describe a finite difference WENO scheme for a system of the form

$$
U_{t}+F(U)_{x}=R(U),
$$

and apply it to the system (4.1) with

$$
\begin{array}{r}
F(U)=\left(v+\mu p(u)_{x}, 0\right)^{T}, \\
R(U)=\left(\mu p(u)_{x x}, \frac{1}{\varepsilon^{2}}\left(q(u)-\left(v+p(u)_{x}\right)\right)\right) .
\end{array}
$$

As $\varepsilon \rightarrow \infty$ and $\mu \rightarrow 0$, the system becomes

$$
\begin{aligned}
u_{t}+v_{x} & =0, \\
v_{t} & =0
\end{aligned}
$$

and the characteristic speed of the system is $\lambda=0$ (twice). As $\varepsilon \rightarrow 0$ and $\mu \rightarrow 1, v+\mu p(u)_{x} \rightarrow q(u)$ and the system relaxes to the equation

$$
u_{t}+q(u)_{x}=p(u)_{x x}
$$


and the characteristic speed of the left hand side is given by $\lambda=q^{\prime}(u)$.

Conservative finite difference for system (4.1) are written as follows, 36.

$$
\frac{d U_{j}}{d t}=-\frac{\hat{F}_{j+\frac{1}{2}}-\hat{F}_{j-\frac{1}{2}}}{\Delta x}+G\left(U_{j}\right)
$$

where $U_{j}(t) \approx U\left(x_{j}, t\right)$ is an approximation of the pointwise value of $U$ at grid nodes, and the numerical flux at cell edge $x_{j+\frac{1}{2}}$ is computed as follows

$$
\hat{F}_{j+\frac{1}{2}}=\hat{F}_{j}^{+}\left(x_{j+\frac{1}{2}}\right)+\hat{F}_{j+1}^{-}\left(x_{j+\frac{1}{2}}\right) .
$$

The function $\hat{F}_{j}^{+}(x)$ and $\hat{F}_{j+1}^{-}(x)$ are suitable reconstructions defined, respectively, in cell $j$ and in cell $j+1$. They are obtained as follows. First, we assume that the flux can be split into a positive and negative component

$$
F(U)=F^{+}(U)+F^{-}(U),
$$

with $\lambda\left(\nabla_{U} F^{+}(U)\right) \geq 0, \lambda\left(\nabla_{U} F^{-}(U)\right) \leq 0$. The quantity $F_{j}^{ \pm}=F^{ \pm}\left(U_{j}\right)$ are computed at cell center. Then $\hat{F}_{j}^{ \pm}(x)$ are reconstructed from $\left\{F_{j}^{ \pm}\right\}$using high order essentially non oscillatory reconstruction, such as ENO or WENO, that allows pointwise reconstruction of a function from its cell averages, (see, e.g. [40] for details).

The flux $F$ may contain derivatives. For example the first equation in system (4.1) contains $p(u)_{x}$. Such terms are computed by point-wise WENO reconstruction.

In all our examples we used the simple local Lax-Friedrix flux decomposition, i.e. $F^{+}(U)=\frac{1}{2}(F(U)+$ $\alpha U), F^{-}(U)=\frac{1}{2}(F(U)-\alpha U), \alpha \geq \max _{U}\left|\rho\left(\nabla_{U} F\right)\right|, \forall A \in \mathbb{R}^{m \times m}$, where $\rho(A)=\max _{1 \leq i \leq m}\left|\lambda_{i}(A)\right|$ denotes the spectrum radius of matrix $A$, and the max defining $\alpha$ is taken for $U$ varying in a suitable range in a neighborhood of each cell. In our test case we chose $\alpha=1$, since as $\varepsilon \rightarrow \infty, \rho\left(\nabla_{U} F\right)=0$ and in our numerical test $q(u)$ is either $0, u$, or $u^{2} / 2$, with $U$ ranging in $[0,1]$, therefore $\left|q^{\prime}(u)\right| \leq 1$.

We remark here that the choice of $\alpha$ is based on the characteristic speeds of the limit convectiondiffusion equation, while a more detailed analysis is needed to justify its use in intermediate regions, for which the characteristic speeds can be much higher, and the stabilization that compensates for the apparent violation of the hyperbolic CFL condition comes from the implicit treatment of the diffusion term.

Furthermore for large value of $\varepsilon$, (e.g., $\varepsilon=1$ ), we want to avoid adding and subtracting terms which may cause loss of accuracy. For a semidiscrete scheme the function $\mu$ will depend also on the grid space $\Delta x$. A simple choice for $\mu$ is given by

$$
\mu=\exp \left(-\varepsilon^{2} / \Delta x\right)
$$

which is what we used in all our numerical tests.

For the diffusion term $p(u)_{x x}$ we used the standard 2-nd order finite difference technique for second order time discretization, and the standard 4-th order finite difference technique where 3-rd order time discretization are used.

5. Numerical examples. In this section we test several second and third order IMEX R-K schemes presented in the literature that satisfy the algebraic order conditions (3.9) and conditions in Definition 3. Usually, IMEX time discretization are identified by an acronym (e.g. the initials of the authors), and three numbers $\left(\sigma_{E}, \sigma_{I}, p\right)$ denoting, respectively, the effective number of stages (in practice the number of function evaluations) of the explicit and implicit scheme and the classical order of accuracy.

Below we list the IMEX R-K schemes used in the numerical tests.

- $\operatorname{SSP}(3,3,2)$ : derived by Pareschi, Russo [36], it is a second order IMER R-K of type A, the explicit part is strongly stability preserving, while the implicit part is stiffly accurate. In accordance with the proposition 8.1 in the Appendix 8.2 this scheme is not globally stiffly accurate according to Definition 3.1

- $\operatorname{ARS}(2,2,2)$ : derived by Asher, Ruuth, Spiteri [1, it is a second order scheme, the double Butcher tableau of this scheme is reproduced in Appendix 8.3. Note that this scheme is globally stiffly accurate according to the Definition 3.1 and satisfies the additional order conditions (3.9). 
- $\operatorname{ARS}(4,4,3)$ : derived by Asher, Ruuth, Spiteri 1, it is a third order scheme, the double Butcher tableau of this scheme is reproduced in Appendix 8.3. Similarly to $\operatorname{ARS}(2,2,2)$ this scheme is globally stiffly accurate according to Definition 3.1 and satisfies the additional order conditions (3.9).

- $\operatorname{BPR}(3,5,3)$ introduced in this paper is a third order IMEX R-K scheme of type CK and globally stiffly accurate according to Definition 3.1. This scheme has $s=5$ internal stages, $\sigma_{E}=3$ explicit stages and $\sigma_{I}=5$ implicit stages. The additional order conditions (3.9) are satisfied. This scheme is more efficient than $\operatorname{ARS}(4,4,3)$ for the explicit part, but less efficient for the implicit one. In many cases the computation of the explicit term is more expensive than the solution of the implicit step, thus resulting in an overall improvement in efficiency per time step. Furthermore, the coefficients of the explicit scheme are all non negative, which is an advantage for the stability properties of the scheme. We reproduced the coefficients of this scheme in Appendix 8.3 .

In all the computations presented in this paper we denote each scheme with an acronym indicating the IMEX scheme and the type of space discretization.

Space discretization is identified by a short name containing the order of accuracy in space; for example, WENO53 (or WENO32), see for details [0], means a fifth (or a third) order reconstruction which reduced to third (or second) order near singularities and CdS2 stands for second order central discretization scheme.

We remark here that all the analysis performed in the paper and the numerical tests are performed under the assumption that the initial data is well-prepared, which means that the initial condition lies in the limit manyfold as $\varepsilon \rightarrow 0$. If this condition is not satisfied, then a loss of accuracy is observed, unless some initial layer fix is adopted. Schemes of type A are more robust against this problem, as is described in [36].

5.1. Convergence test. In this section we investigate numerically the convergence rate of the second and third IMEX R-K schemes introduced before for a wide range of the parameter $\varepsilon$. To this aim we apply these schemes to simple prototype hyperbolic system (3.1), with initial conditions chosen in such a way that the exact solutions is smooth and does not present a rapidly varying transient for small values of $\varepsilon$. This is achieved in practice by imposing that the initial condition satisfies the limit relation between $u$ and $v$ as $\varepsilon \rightarrow 0$.

Numerical convergence rate is calculated by the formula

$$
p=\log _{2}\left(E_{\Delta t_{1}} / E_{\Delta t_{2}}\right),
$$

where $E_{\Delta t_{1}}$ and $E_{\Delta t_{2}}$ are the global errors computed with step $\Delta t_{1}=\mathcal{O}(\Delta x)$, and $\Delta t_{2}=\Delta t_{1} / 2$. In the following tests we put $\varepsilon^{2}=10^{-6}$ and we choose $\Delta t \approx \Delta x$.

For the first test we set $p(u)=u$ and $q(u)=0$. Then we get

$$
\begin{aligned}
& u_{t}=-v_{x}-\mu u_{x x}+\mu u_{x x}, \\
& \varepsilon^{2} v_{t}=-u_{x}-v,
\end{aligned}
$$

that in the limit case, $\varepsilon=0$ and $\mu=1$ leads to the linear diffusive problem

$$
u_{t}=u_{x x}, \quad u(x, 0)=u_{0}(x) .
$$

We use periodic boundary conditions with $u_{0}(x)=\cos (x)$, and $x \in[0,2 \pi]$, so that $u(x, t)=u_{0}(x) \exp (-t)$ is an exact solution of (5.2). The final time is $T=1$ and $\Delta t=0.5 \Delta x$.

The results are reported in Table 5.1 and 5.2 showing that the expected convergence rates are reached for the $u$-component.

Next we set $p(u)=q(u)=u$ and consider the following system

$$
\begin{aligned}
u_{t}+v_{x} & =\mu u_{x x}-\mu u_{x x} \\
\varepsilon^{2} v_{t}+u_{x} & =-(v-u),
\end{aligned}
$$

where the limiting behavior is given by an advection-diffusion equation. We use periodic boundary conditions with the initial data $u(x, 0)=\exp (-(1+\cos (x-\pi)) / \sigma), v(x, 0)=u(x, 0)(1-\mu \sin (x-\pi) / \sigma)$ 
TABLE 5.1

Convergence rate for $u$ in $L_{\infty}$-norm.

\begin{tabular}{|c|c|c|c|c|}
\hline \multicolumn{2}{|c|}{$\begin{array}{c}\operatorname{ARS}(2,2,2)-\mathrm{CdS} 2 \\
e_{s}^{T} \tilde{A}=\tilde{b}^{T}\end{array}$} & $\begin{array}{c}\mathrm{SSP} 2(3,3,2)-\mathrm{CdS} 2 \\
e_{s}^{T} \tilde{A} \neq \tilde{b}^{T}\end{array}$ & $\begin{array}{c}\operatorname{ARS}(2,2,2)-\text { WENO32 } \\
e_{s}^{T} \tilde{A}=\tilde{b}^{T}\end{array}$ & $\begin{array}{c}\text { SSP2 }(3,3,2)-W E N O 32 \\
e_{s}^{T} \tilde{A} \neq \tilde{b}^{T}\end{array}$ \\
\hline & $L_{\infty}(u)$ Order & $N \quad L_{\infty}(u)$ Order & $N \quad L_{\infty}(u)$ Order & $N \quad L_{\infty}(u)$ Order \\
\hline & 7.8 & 202. & 204. & 20 \\
\hline & 42 & 31.86 & 401. & 66 \\
\hline 80 & $4.597 \mathrm{e}-042.02$ & 31.96 & 804. & 804 \\
\hline 160 & 42 & 41.99 & 1601 & .93 \\
\hline 320 & $2.833 \mathrm{e}-052.00$ & $3201.274 \mathrm{e}-042.00$ & $320 \quad 2.760 \mathrm{e}-05 \quad 1.97$ & $3202.748 \mathrm{e}-05 \quad 1.96$ \\
\hline
\end{tabular}

TABLE 5.2

Convergence rate for $u$ in $L_{\infty}$-norm.

\begin{tabular}{|c|c|}
\hline $\begin{array}{c}\text { ARS(4,4,3)-WENO53 } \\
e_{s}^{T} \tilde{A}=\tilde{b}^{T}\end{array}$ & $\begin{array}{c}\text { BPR }(5,5,3)-\text { WENO53 } \\
e_{s}^{T} \tilde{A}=\tilde{b}^{T}\end{array}$ \\
$N \quad L_{\infty}(u)$ Order & $N \quad L_{\infty}(u)$ Order \\
\hline \hline $201.810 \mathrm{e}-02$ & $201.639 \mathrm{e}-02$ \\
$403.365 \mathrm{e}-032.42$ & $403.099 \mathrm{e}-032.40$ \\
$80.349 \mathrm{e}-042.65$ & $805.167 \mathrm{e}-042.58$ \\
$1605.960 \mathrm{e}-053.16$ & $1605.821 \mathrm{e}-053.14$ \\
$3205.968 \mathrm{e}-06 \quad 3.31$ & $3205.949 \mathrm{e}-063.29$ \\
\hline
\end{tabular}

with $\sigma=0.05$ and $\mu=1$, on the spatial interval $[0,2 \pi]$, at the final time $T=0.3$ anfd $\Delta t=0.5 \Delta x$. As reference solution we use the truncated Fourier representation of the exact solution

$$
U_{e x a}(x, t)=\sum_{k=-\infty}^{+\infty} U_{k}(t) e^{i k x}, \quad V_{\text {exa }}(x, t)=\sum_{k=-\infty}^{+\infty} V_{k}(t) e^{i k x}
$$

with $U_{k}(t)$ and $V_{k}(t)$ satisfying

$$
\begin{aligned}
& \dot{U}_{k}=-i k V_{k}, \\
& \varepsilon^{2} \dot{V}_{k}=-i k U_{k}+U_{k}-V_{k} .
\end{aligned}
$$

For each $k$, system (5.3) can be written as a $2 \times 2$ constant coefficient homogeneous system which can be solved exactly. The results are given in Table 5.3 showing that again the expected convergence rates are reached for the $u$-component by all schemes.

The above convergence analysis has been performed in the limit $\varepsilon \rightarrow 0$, therefore we might expect a degradation of the accuracy for intermediate regimes as in the case of hyperbolic relaxation when the classical order is greater then two [5, 8, 11. Furthermore, from the practical point of view, the understanding of this phenomenon is essential in situations where one is interested in the construction of higher order methods.

Figure 5.1 shows the convergence rates as a function of $\varepsilon^{2}$ using different values of $\varepsilon^{2}$ ranging from $10^{-6}$ to 1 and $\Delta t \simeq \Delta x$. Second order schemes ARS(2,2,2)-CdS2, SSP2(3,3,2)-CdS2 have the prescribed order of accuracy uniformly in $\varepsilon^{2}$ (upper left panel). Instead, ARS(2,2,2)-WENO32 and SSP2(3,3,2)WENO32 present a degradation of accuracy at intermediate regimes (upper right panel).

A similar lack of convergence in intermediate regimes is observed for both the third order schemes ARS(4,4,3)-WENO53 and BPR(3,5,3)-WENO53 (lower left panel). This results have a very different nature than the accuracy degradation observed in IMEX schemes applied to hyperbolic systems with stiff relaxation [5]. A plausible reason here appears to be that in intermediate regimes the two terms which are added and subtracted in the equations, i.e. $\pm \mu p(u)_{x x}$, are discretized in two very different ways: one is computed inside the flux (ARS(4,4,3)-WENO53 and BPR(3,5,3)-WENO53), and the other one $\operatorname{ARS}(4,4,3)$-WENO53* and $\operatorname{BPR}(3,5,3)$-WENO53*) is discretized by a discrete one dimensional Laplacian, therefore the two terms do not almost cancel each other. Although in certain regimes such problem could be solved by treating the term $p(u)_{x}$ out of the flux (see, for example, the result in the lower right panel of Figure 5.1 for $\operatorname{BPR}(3,5,3))$ and discretizing both terms $\pm \mu p(u)_{x x}$ in the same way, this may compromise the cancelation of the quantity $q(u)-v-p(u)_{x}$ in the flux. A general understanding 
TABLE 5.3

Convergence rate for $u$ in $L_{\infty}$-norm in the convection-diffusion limit.

\begin{tabular}{|c|c|c|c|}
\hline $\begin{array}{c}\text { ARS(2,2,2)-CdS2 } \\
e_{s}^{T} \tilde{A}=\tilde{b}^{T}\end{array}$ & $\begin{array}{c}\text { SSP2(3,3,2)-CdS2 } \\
e_{s}^{T} \tilde{A} \neq \tilde{b}^{T}\end{array}$ & $\begin{array}{c}\text { ARS(4,4,3)-WENO53 } \\
e_{s}^{T} \tilde{A}=\tilde{b}^{T}\end{array}$ & $\begin{array}{c}\text { BPR(5,5,3)-WENO53 } \\
e_{s}^{T} \tilde{A}=\tilde{b}^{T}\end{array}$ \\
$N L_{\infty}(u)$ Order & $N L_{\infty}(u)$ Order & $N L_{\infty}(u)$ Order & $N L_{\infty}(u)$ Order \\
\hline \hline $403.867 \mathrm{e}-03$ & $402.615 \mathrm{e}-03$ & $404.297 \mathrm{e}-04$ & $408.300 \mathrm{e}-04$ \\
$809.457 \mathrm{e}-042.03$ & $806.243 \mathrm{e}-042.06$ & $805.770 \mathrm{e}-052.89$ & $801.167 \mathrm{e}-042.83$ \\
$1602.330 \mathrm{e} 042.02$ & $1601.543 \mathrm{e}-042.01$ & $1607.922 \mathrm{e}-062.86$ & $1601.603 \mathrm{e}-052.86$ \\
$3205.798 \mathrm{e}-052.00$ & $3203.850 \mathrm{e}-052.00$ & $3201.256 \mathrm{e}-062.65$ & $3202.230 \mathrm{e}-062.85$ \\
\hline
\end{tabular}

and a robust treatment of intermediate regimes is beyond the scope of the present paper and requires further investigation.
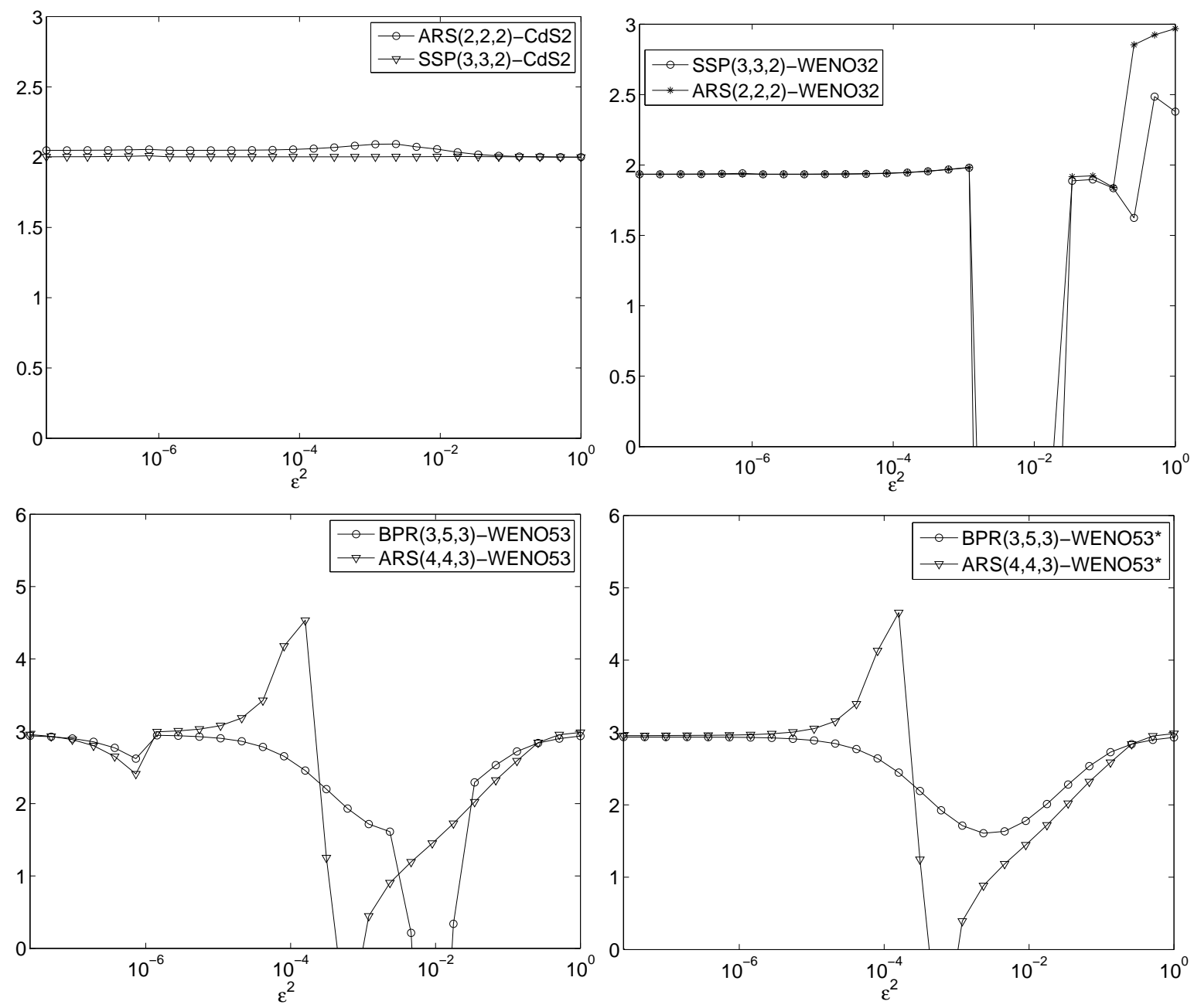

FIG. 5.1. Convergence rate in $L_{\infty}$-norm versus $\varepsilon^{2}$ for various schemes. Lack of convergence for intermediate values of $\varepsilon$ is evident in the upper right and lower left panels. The lower right panel shows results obtained by a scheme in which the explicit and implicit term $\mu p(u)_{x x}$ is discretized spatially in identical way, namely by a second order discrete Laplacian.

5.2. Shock test cases. In this section we apply the scheme to problems with discontinuous initial data, that in the limit as $\varepsilon \rightarrow 0$ reduce to convection-diffusion equation. Notice that in the relaxed limit the scheme becomes an IMEX scheme for the limit equation. This test is used to check both the shock capturing properties of the scheme, and its relaxation to an IMEX scheme for the limit equation.

In the rest of the section we will consider the third order $\operatorname{BPR}(3,5,3)$-WENO53 scheme. 

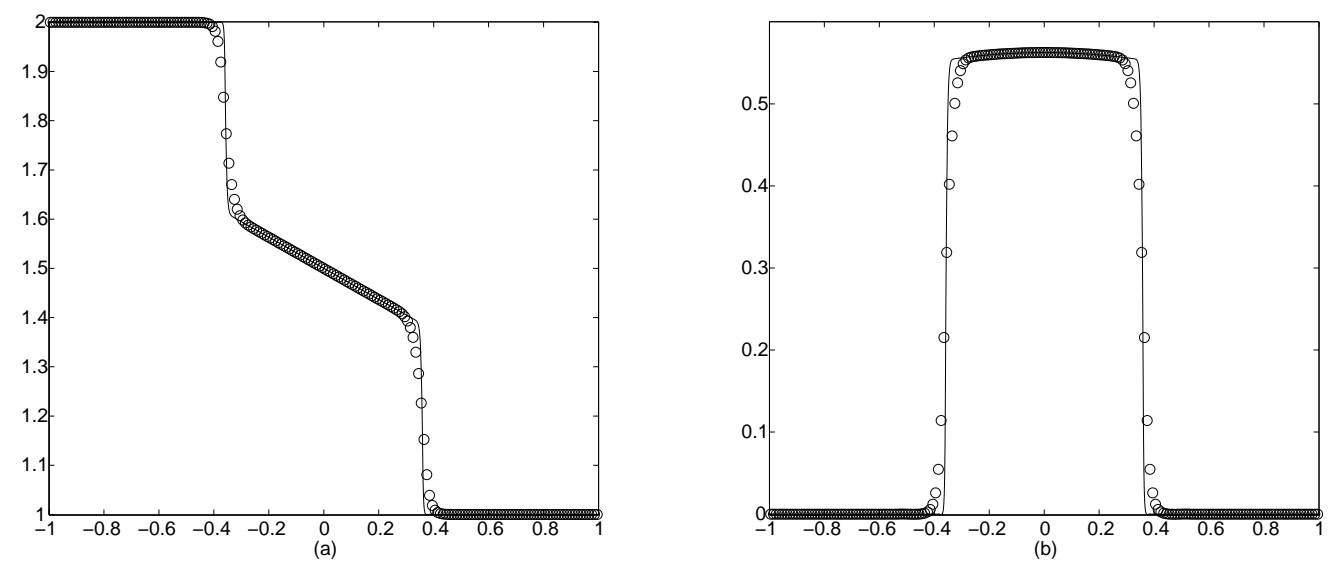

FiG. 5.2. Numerical solutions at time $t=0.25$ in the rarefied regime $\left(\varepsilon^{2}=0.7\right)$ with $\Delta t=0.5 \Delta x$ and $\Delta x=0.01$. On the left-hand side the mass density $u(a)$ and on the right-hand side the flow $v(b)$. Solid line is the reference solution.

First we consider a purely diffusive linear problem. We solve a Riemann problem, in the rarefied and diffusive regime for system

$$
\begin{aligned}
u_{t}+v_{x} & =\mu u_{x x}-\mu u_{x x} \\
\varepsilon^{2} v_{t}+u_{x} & =-v .
\end{aligned}
$$

We take the following initial data

$$
\begin{array}{ll}
u_{L}=2.0 & v_{L}=0, \quad-1<x<0 \\
u_{R}=1.0 & v_{R}=0, \quad 0<x<1 .
\end{array}
$$

As $\varepsilon$ goes to zero we get $u_{t}=u_{x x}$, i.e. the problem becomes a classical Riemann problem for the heat equation.

In order to test our scheme we compute the numerical solution in the rarefied $\left(\varepsilon^{2}>\Delta x\right)$ regime and in the diffusive $\left(\varepsilon^{2} \ll \Delta x\right)$ regime. This means that when $\varepsilon^{2}$ is very large (i.e., rarefied regime) $\mu$ is very small, and on the other hand when $\varepsilon$ is very small (i.e., diffusive regime) $\mu$ is equal to 1 .

We set $\varepsilon^{2}=0.7$ for the rarefied regime and $\varepsilon^{2}=10^{-6}$ for the diffusive regime (or stiff regime). The numerical solution for $u$ and $v$ in the rarefied (Fig 5.2) and diffusive regime (Fig 5.3) are depicted with a reference solution obtained using a fine spatial grid of $N=2000$ cells.

As boundary conditions we set $v=0$ at $x= \pm 1$. Compatibility with the system gives $u_{x}=0$ at $x= \pm 1$. Notice that the characteristic variables for this problem are $\xi_{ \pm}=u \pm \varepsilon v$, therefore condition $v=0$ at $x= \pm 1$ is equivalent to impose $\xi_{+}=\xi_{-}$at the boundary. For such a reason we denote these boundary conditions as reflecting boundary conditions.

The solution is reported at final time $t=0.25$ in the rarefied regime (Fig. 5.2) and $t=0.04$ in the diffusive regime (Fig. 5.3). In the figures we observe that the scheme captures well the correct behavior of the solutions both in rarefied regime where it provides an accurate description of the shock without oscillations near the discontinuities, and in the diffusive regime where the numerical solution matches accurately the reference solution.

Finally we consider the nonlinear Ruijgrok-Wu model, [38, (for details see 25])

$$
\begin{aligned}
u_{t}+v_{x} & =\mu(\varepsilon) \frac{u_{x x}}{2 k_{0}}-\mu(\varepsilon) \frac{u_{x x}}{2 k_{0}} \\
\varepsilon^{2} v_{t}+u_{x} & =-2 k_{0}\left[v-\frac{C}{2}\left(u^{2}-\varepsilon^{2} v^{2}\right)\right]
\end{aligned}
$$



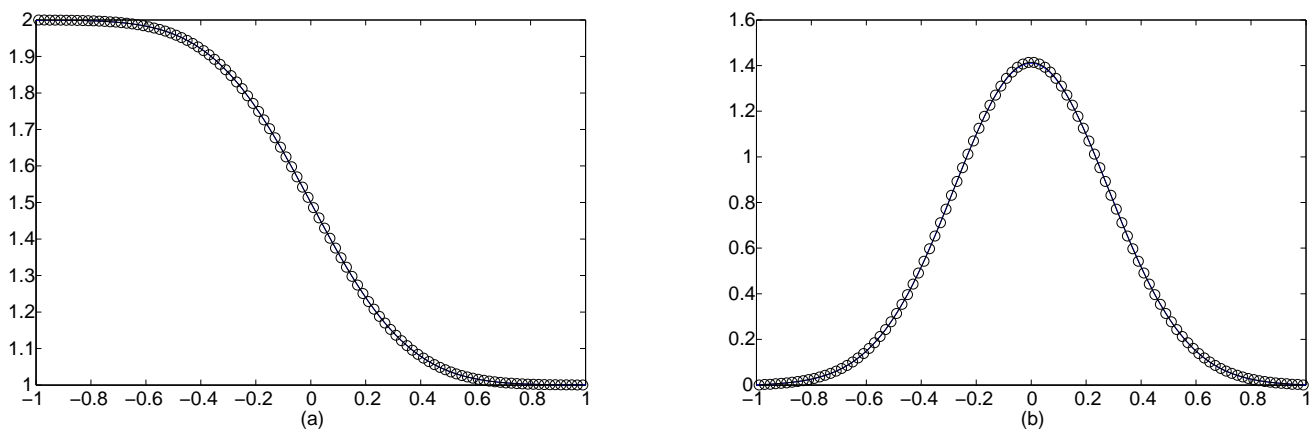

FIG. 5.3. Numerical solutions at time $t=0.04$ in the parabolic regime $\left(\varepsilon^{2}=10^{-6}\right)$ with $\Delta t=0.5 \Delta x$ and $\Delta x=0.02$. On the left-hand side the mass density (a) $u$ and on the right-hand side the flow $v(b)$. Solid line is the reference solution.
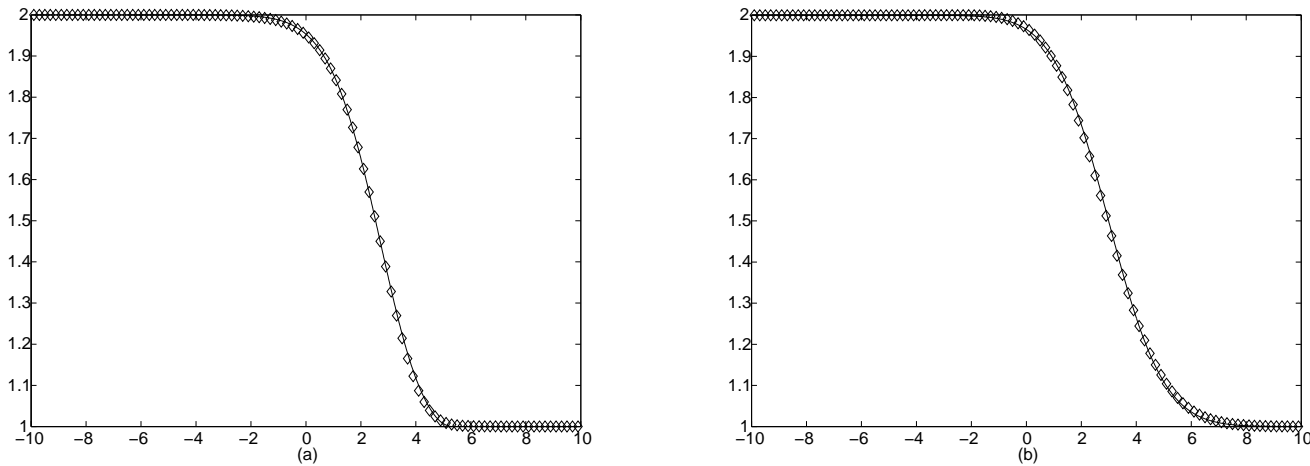

FIG. 5.4. Numerical solutions at time $t=2.0,(a)$ in the intermediate regime $\left(\varepsilon^{2}=0.4\right.$ ) and (b) parabolic regime $\left(\varepsilon^{2}=10^{-6}\right)$ with $\Delta t=0.25 \Delta x$ and $\Delta x=0.2$. Solid line is the exact solution.

where we added and subtracted to the original model the quantity $\mu u_{x x}$ on the right-hand side of the first equation and $C$ is a constant, (in our test we chose $C=1$ ). In the diffusive limit $\varepsilon \rightarrow 0$, the second equation provides

$$
v=\frac{1}{2} u^{2}-\frac{1}{2 k_{0}} u_{x}
$$

and we get the limiting viscous Burgers equation

$$
u_{t}+\left(\frac{u^{2}}{2}\right)_{x}=\frac{u_{x x}}{2 k_{0}}
$$

Note that through BPR approach the IMEX R-K scheme in the diffusive limit relaxes to the same IMEX R-K one for equation (5.5) where the convection term is treated explicitly and the diffusion term implicitly.

The exact solution to the shock-wave problem has been given in 38. The initial conditions are two local Maxwellians characterized by

$$
\begin{gathered}
u_{L}=2.0, \quad-10<x<0, \\
u_{R}=1.0, \quad 0<x<10,
\end{gathered}
$$

with $v=\left[\left(1+u^{2} \varepsilon^{2}\right)^{1 / 2}-1\right] / \varepsilon^{2}$.

In Figure 5.4 we show the computed solution for the mass density $u$ in the intermediate $\left(\varepsilon^{2}=0.4\right)$ and parabolic $\left(\varepsilon^{2}=10^{-6}\right)$ regimes versus the exact solution. As it can be seen once again, the scheme gives an accurate description of the viscous shock profiles. 
6. Application to transport equations. In this section we apply the IMEX schemes derived in the first part of the manuscript to the case of neutron transport equations [28, 26, 32.

We consider the multidimensional transport equation under the diffusive scaling. Let $f(t, \mathbf{x}, \mathbf{v})$ be the probability density distribution for particles at space point $\mathbf{x} \in \mathbb{R}^{d}$, time $t$, traveling with velocity $\mathbf{v} \in \Omega \subset \mathbb{R}^{d}$ with $\int_{\Omega} d \mathbf{v}=S$. Here $\Omega$ is symmetric in $\mathbf{v}$, meaning that $\int_{\Omega} g(\mathbf{v}) d \mathbf{v}=0$ for any function $g$ odd in $\mathbf{v}$. Then $f$ solves the non dimensional linear transport equation

$$
\varepsilon \partial_{t} f+\mathbf{v} \nabla_{x} f=\frac{1}{\varepsilon}\left(\frac{\sigma_{s}}{S} \int_{\Omega} f d \mathbf{v}^{\prime}-\sigma f\right)+\varepsilon Q,
$$

where $\sigma=\sigma(x)$ is the total cross section, $\sigma_{s}=\sigma_{s}(x)$ is the scattering coefficient. Here $Q=Q(x)$ is a source term and $\varepsilon$ the mean free path. Typically, $\sigma_{s}=\sigma-\varepsilon^{2} \sigma_{A}$ where $\sigma_{A}=\sigma_{A}(x)$ is the absorption coefficient. Such an equation arises in neutron transport [12, radiative transfer [14] and wave propagation in random media 39, etc. In all these applications, the scaling appearing in (6.1) is typical, and gives rise to a diffusion equation as $\varepsilon \rightarrow 0$ of the form [26]

$$
\partial_{t} \rho=\frac{1}{S} \int_{\Omega} \mathbf{v} \cdot \nabla_{x}\left(\frac{\mathbf{v}}{\sigma} \cdot \nabla_{x} \rho\right) d \mathbf{v}-\sigma_{A} \rho+Q,
$$

where $\rho=(1 / S) \int_{\Omega} f d \mathbf{v}$. We refer, for example, to [4, 2] and the references therein for rigorous mathematical results concerning diffusion limits of transport equations.

6.1. Problem reformulation. Consider now the one-dimensional transport equation

$$
\varepsilon \partial_{t} f+v \partial_{x} f=\frac{1}{\varepsilon}\left(\frac{\sigma_{s}}{2} \int_{-1}^{1} f d v^{\prime}-\sigma f\right)+\varepsilon Q,
$$

with $x_{L}<x<x_{R}$ and boundary conditions

$$
\begin{gathered}
f\left(t, x_{L}, v\right)=F_{L}(v), \quad \text { for } v>0, \\
f\left(t, x_{R},-v\right)=F_{R}(v), \quad \text { for } v>0 .
\end{gathered}
$$

In 26] the authors proposed a method based on the even-odd decomposition $f=r+\varepsilon j$ where $r=$ $\frac{1}{2}(f(v)+f(-v))$ and $j=\frac{1}{2 \varepsilon}(f(v)-f(-v))$, that splits the equation (6.2) as two equations, each for $v>0$

$$
\begin{array}{r}
\varepsilon \partial_{t} f(v)+v \partial_{x} f(v)=\frac{1}{\varepsilon}\left(\frac{\sigma_{s}}{2} \int_{-1}^{1} f d v^{\prime}-\sigma f(v)\right)+\varepsilon Q, \\
\varepsilon \partial_{t} f(-v)-v \partial_{x} f(-v)=\frac{1}{\varepsilon}\left(\frac{\sigma_{s}}{2} \int_{-1}^{1} f d v^{\prime}-\sigma f(-v)\right)+\varepsilon Q .
\end{array}
$$

Adding and subtracting these two equations leads to

$$
\begin{array}{r}
\partial_{t} r+v \partial_{x} j=-\frac{\sigma_{s}}{\varepsilon^{2}}(r-\rho)-\sigma_{A} r+Q \\
\partial_{t} j+\frac{v}{\varepsilon^{2}} \partial_{x} r=-\frac{\sigma_{s}}{\varepsilon^{2}} j-\sigma_{A} j,
\end{array}
$$

where

$$
\rho=\int_{0}^{1} r d v
$$

As $\varepsilon \rightarrow 0$, system (6.5) gives

$$
r=\rho, \quad j=-(v / \sigma) \partial_{x} r .
$$

Applying this to the first equation of (6.5) and integrating over $v$ we get the diffusion equation

$$
\partial_{t} \rho=\frac{1}{3} \partial_{x x} \rho-\sigma_{A} \rho+Q
$$


To get boundary conditions for $r$ and $j$ we use relations

$$
r+\left.\varepsilon j\right|_{x=x_{L}}=F_{L}(v), \quad r-\left.\varepsilon j\right|_{x=x_{R}}=F_{R}(v) .
$$

For arbitrary value of $\varepsilon$, the compatibility conditions imposed at the boundary is quite complicated. However, for small values of $\varepsilon$, which is the case we are interested in, the treatment simplifies, because when $\varepsilon \rightarrow 0, j=-(v / \sigma) \partial_{x} r$, then applying this in (6.8) one gets

$$
r-\left.\varepsilon v \partial_{x} r\right|_{x=x_{L}}=F_{L}(v), \quad r+\left.\varepsilon v \partial_{x} r\right|_{x=x_{R}}=F_{R}(v) .
$$

Such boundary conditions will avoid a boundary layer in the limit case $\varepsilon \rightarrow 0$, therefore the numerical boundary conditions are obtained by discretizing Eq. (6.9). As done in [26], the boundary conditions have been applied using a second order implementation of equation (6.8) based on central differences. Extensions to higher-order implementation of equations (6.9) and to different boundary conditions are not considered here and will be investigated in a forthcoming work. According to this, for our tests we chose a second order scheme in space and time, because the boundary conditions are discretized to second order accuracy.

Now we start from system (6.5) and, adding and subtracting the quantity $v^{2} \partial_{x x} r / \sigma$ in the first equation, we reformulate the problem in the equivalent form

$$
\begin{aligned}
& \partial_{t} r=\underbrace{-v \partial_{x}\left(j+\frac{\mu(\varepsilon) v \partial_{x} r}{\sigma}\right)}_{\text {Explicit }} \underbrace{-\frac{\sigma_{s}}{\varepsilon^{2}}(r-\rho)-\sigma_{A} r+Q+\mu(\varepsilon) v^{2} \frac{\partial_{x x} r}{\sigma}}_{\text {Implicit }}, \\
& \partial_{t} j=\underbrace{-\frac{1}{\varepsilon^{2}}\left(j+\frac{v \partial_{x} r}{\sigma}\right)}_{\text {Implicit }},
\end{aligned}
$$

The new system is then discretized in time using an IMEX R-K scheme as described in the first part of the manuscript. The implicit-explicit integration process is emphasized in (6.10).

We remark that this new formulation of the diffusive relaxation system (6.5) is such that when $\varepsilon$ tends to zero the system relaxes towards (6.7). From a numerical point of view the new formulation has several advantages. In particular, as $\varepsilon \rightarrow 0$, the IMEX R-K scheme applied to system (6.10) originates a fully implicit scheme for solving the diffusion equation (6.7).

In our numerical tests in order to obtain uniformly accurate second order scheme both in space and in time for the BPR approach, we consider SSP2(3,3,2)-WENO32. We remark that we can obtain analogous results considering $\operatorname{ARS}(2,2,2)$-WENO32.

The equations are discretized in space and velocity, i.e. $r\left(x_{i}, v_{m}, t_{n}\right) \approx r_{i, m}^{n}$ where $\left\{v_{m}\right\}$ are chosen to be the $N_{v}$ positive nodes of the Gauss-Legendre quadrature formula, with $2 N_{v}$ nodes in the interval $[-1,1]$ while $x_{i}=\Delta x(i-1 / 2)$ for $i=1, \ldots, N_{p}$. Note that the computation of the $k$-th stage of the implicit equation $r_{i, m}^{(k)}$, requires the quantity $\rho_{i}^{(k)}$ in the implicit part in (6.10). Such quantities are obtained as follows. Assume we have computed $r_{i, m}^{(l)}$ for $l=1, \ldots, k-1$, then $r_{i, m}^{(k)}$ is obtained from

$$
r_{i, m}^{(k)}=\bar{r}_{i, m}^{(k-1)}+\Delta t a_{k k}\left(\frac{\sigma_{S}}{\varepsilon^{2}}\left(r_{i, m}^{(k)}-\rho_{i}^{(k)}\right)-\sigma_{A} r_{i, m}^{(k)}+Q\right)
$$

discretizing system (6.10) without the quantity $\mu(\varepsilon) v^{2} \partial_{x x} r / \sigma$ in the implicit and explicit part. The quantity $\bar{r}_{i, m}^{(k-1)}$ represents the contribution of the first $k-1$ stages. Then, in order to compute $\rho_{i}^{(k)}$ we apply Gauss quadrature on both sides of (6.11) (i.e. multiply by the weights $w_{m}$ and sum over $m$ ), setting $\mu=0$. In this way we obtain an equation for $\rho_{i}^{(k)}$ that can be explicitly solved, and such value is plugged in (6.10) in order to compute $r_{i, m}^{(k)}$.

6.2. Numerical results. In this section we shall consider some transport problems in slab geometry. We will present the transient and the steady state solutions. We remark that in all the test problems we have used $N_{v}=8$ thus the standard 16 points Gaussian quadrature set for the velocity space. In all the tests the initial distribution is $f(\mathbf{x}, \mathbf{v}, t=0)=0$.

We emphasize that, besides uniform accuracy in $\varepsilon$, our approach allows to choose larger time steps, since there is no stability restriction on the time step. As we will show, this permits to obtain numerical 
results at a lower computational cost compared to other approaches presented in the literature that lead to explicit schemes for the underlying diffusion limit with a parabolic CFL stability restriction [26, 28]. Nevertheless, in order to get an accurate resolution of the behavior of the solution, smaller time step may be necessary.

Depending on the regime of the parameter $\varepsilon$, we compare numerical solutions to a direct implicit discretization of the diffusion limit (6.7) when $\varepsilon$ tends to zero, whereas for intermediate values of the parameter $\varepsilon$ we compute a reference solution using a much finer grid in space.

In the next tests we compare the results obtained by the new approach versus the results given by Jin et al., in [26], here denoted by JPT. We refer to [26, 28, 32] for similar results where the limiting scheme is explicit and so in diffusive regions requires $\Delta t \approx(\Delta x)^{2}$. In all figures we use notations $N_{s}$ and $N_{p}$ to denote the number of time steps and grid points in space respectively.

Problem I:

$$
\begin{array}{ccc}
x \in[0,1], & F_{L}(v)=1, & F_{R}(v)=0, \\
\sigma_{S}=1, \quad \sigma_{A}=0, & Q=0, & \varepsilon=10^{-8} .
\end{array}
$$

The numerical results are reported in Figures 6.1 and 6.2 (a) at different times $t=0.01,0.05,0.15$ with $N_{p}=40$, and at $t=2$ with $N_{p}=20$ where the steady state is reached. In this problem we see that in both cases, the results in the transient and steady state solutions show a good behavior with the correct diffusion limit. The exact diffusive solution has been computed by (6.7) with $N_{p}=200$. As expected both JPT and BPR results are very close to the exact diffusive solution at any times. Note however that thanks to the better stability properties in this regime BPR scheme is about 4 times faster then the explicit method.

Problem II: This is a two-material problem used in [26, 32, 28, where in the purely absorbing region $[0,1]$ the solution decays exponentially whereas in the purely scattering region $[1,11]$ the solution is diffusive, the parameters are the following

$$
\begin{array}{r}
x \in[0,11], \quad F_{L}(v)=5, \quad F_{R}(v)=0, \\
\sigma_{S}=0, \quad \sigma_{A}=1, \quad Q=0, \quad \varepsilon=1, \text { for } x \in[0,1], \\
\sigma_{S}=1, \quad \sigma_{A}=0, \quad Q=0, \quad \varepsilon=0.01, \text { for } x \in[1,11] .
\end{array}
$$

An interface layer is produced between the pure absorbing region and the scattering one. Two meshes are used in the domain $[0,11]$, a thin mesh $\Delta x=0.05$ in $[0,1]$ and a coarse mesh $\Delta x=1$ in $[1,11]$, which means that between the interface layer we have to use a space discretization with a non uniform mesh. Finite volume, rather than finite difference, is used in this case. Since we restrict to second order accuracy, the point-wise values of the source is identified with its cell average. The high order non oscillatory reconstruction is performed by a WENO approach for non uniform mesh. For our numerical tests we used WENO32 reconstruction.

At time $t=150$ the solution has reached the steady state and the results are presented in Figure 6.2 We computed the reference solution with a very fine discretization $N_{p}=400$ using a uniform mesh in all the domain $[0,11]$. The numerical schemes provide a good description for the solution in the absorption and diffusive regions, in fact, we observe that for the steady state, Figure 6.2 (b), JPT and BPR results are close o the reference solution, except at the interface where a slight difference is observed. In this case BPR scheme is about twice times faster then JPT approach.

Problem III: Concerning this problem we present two different situations (see [26, 32]) with nonisotropic boundary conditions that generate a boundary layer:

$$
\begin{gathered}
x \in[0,1], \quad F_{L}(v)=v, \quad F_{R}(v)=0, \\
\sigma_{S}=1, \quad \sigma_{A}=0, \quad Q=0, \quad \varepsilon=10^{-2} .
\end{gathered}
$$

First in an intermediate regime with $\varepsilon=10^{-2}$ and then in a more diffusive regime with $\varepsilon=10^{-4}$. Using a coarse discretization $N_{p}=25$ the boundary layer is not resolved, but we observe that the two approaches accurately capture the solution inside the domain (in Figure (6.3) we have restricted the numerical and the reference solution to the interval $[0,0.5])$. The reference solution has been obtained using a fine 

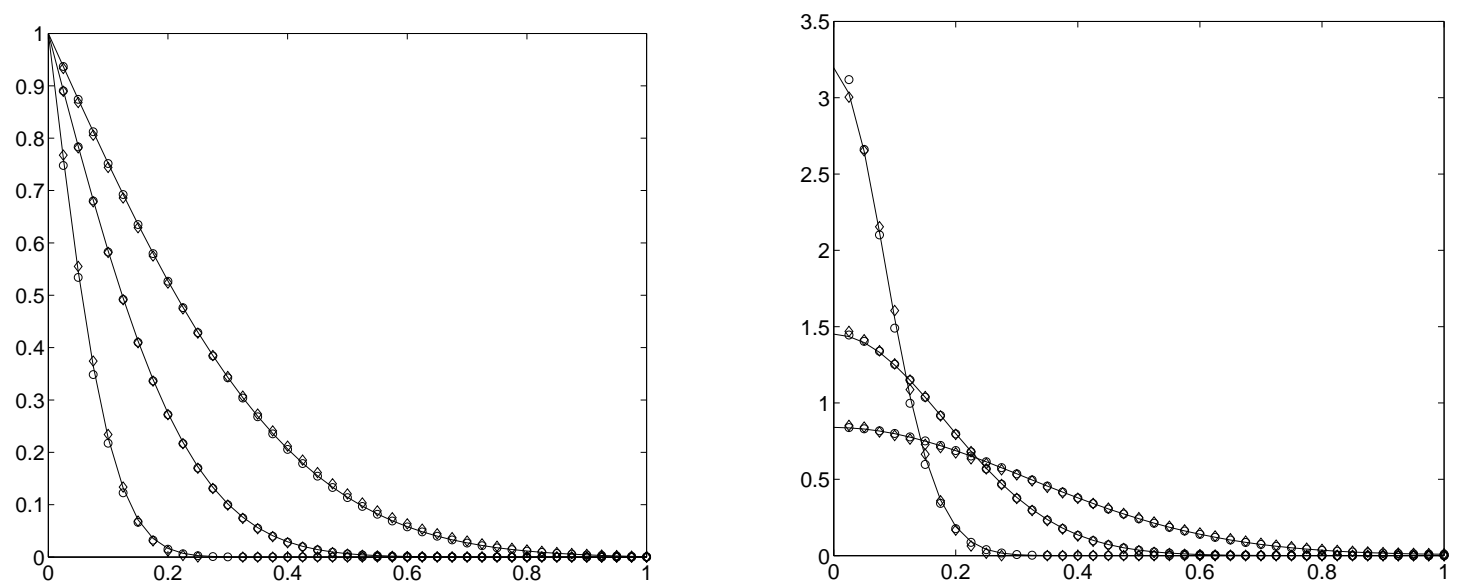

FIG. 6.1. Problem I. On the left-hand side the mass density $\rho$, on the right-hand side the flux $\rho u$. JPT (o), $\Delta x=0.025$, $\Delta t=0.0002, N_{s}=50,250$, 750. BPR $(\diamond), \Delta t=\lambda \Delta x$, with $\lambda=0.035, N_{s}=11,57,171$. The exact diffusive solution is represented by the solid line.

discretization $N_{p}=400$ and the boundary layer is resolved. The results are plotted at time $t=0.4$ in figure 6.3. The higher efficiency of the present method results in an improved time step ratio of a factor 4 .

FIG. 6.2. The steady state solution for the Problem I and II.

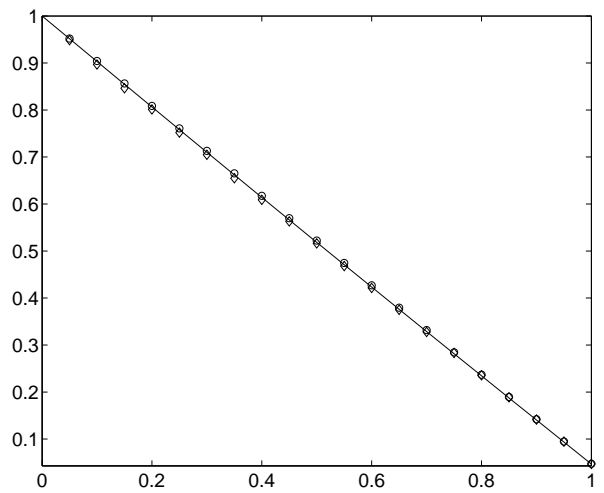

(a) Problem I. The steady state solution of the mass density $\rho$. JPT (o), $\Delta t=0.001, N_{s}=2000 . \operatorname{BPR}(\diamond)$, $\Delta t=0.0035, N_{s}=561$. The exact diffusive solution is represented by the solid line.

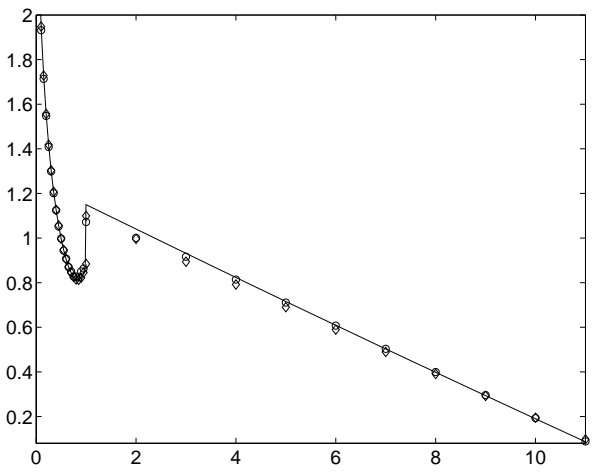

(b) Problem II. The steady state solution of the mass density $\rho$ with $\varepsilon=1$ and $\Delta x=0.05$ on $(0,1)$ and with $\varepsilon=0.01$ and $\Delta x=1$ on $(1,11)$. JPT (o), with $\Delta t=$ 0.025 and $N_{s}=6000$. BPR $(\diamond)$, with $\Delta t=\Delta x$, with $N_{s}=3000$

7. Conclusions. In this manuscript we have presented a general way to tackle diffusion limit for hyperbolic and kinetic problems which permits to obtain accurate and efficient schemes both in rarefied and diffusive regimes. The new approach, in particular, give rise to a fully implicit method for the diffusion component of the limiting system. This is obtained without solving nonlinear systems of implicit equations but by a suitable blending into the IMEX R-K method of a fully implicit solver for the limiting diffusive system. Numerical results show that this approach is able to capture the correct asymptotic behavior of the system at a lower computational cost compared to other approaches that lead to explicit schemes for the underlying diffusion limit, because we removed the parabolic CFL restriction, common to most approaches in the literature. 

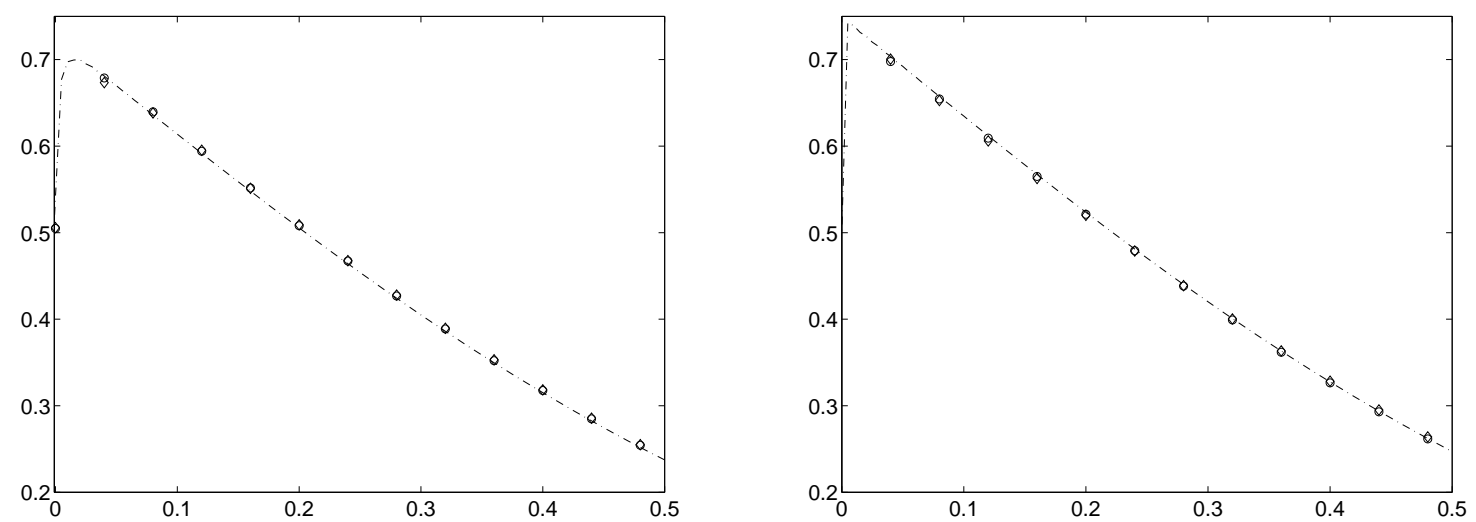

FIG. 6.3. Problem III. The mass density $\rho$. JPT (O), with $\varepsilon=10^{-2}, \Delta x=0.04, \Delta t=0.001, N_{p}=25, N_{s}=400$. $B P R(\diamond), \Delta x=0.04, \Delta t=0.002, N_{p}=25, N_{s}=100$. Left $\varepsilon=10^{-2}$, right $\varepsilon=10^{-4}$. The reference solutions are represented by the dash dot line.

The method here presented is based on the use of IMEX Runge-Kutta methods, however extension to more general additive Runge-Kutta schemes are naturally possible. In fact from our problem reformulation

$$
\begin{aligned}
& u^{\prime}=\underbrace{f_{1}(u, v)}_{\text {explicit }}+\underbrace{f_{2}(u)}_{\text {implicit }}, \\
& \varepsilon^{2} v^{\prime}=\underbrace{g(u, v)}_{\text {implicit }}
\end{aligned}
$$

we can clearly combine different implicit solvers to tackle the "highly" stiff component $g(u, v)$ which originates the algebraic condition $g(u, v)=0$ giving rise to the equilibrium projection $v=G(u)$ and the "mildly" stiff component $f_{2}(u)$ corresponding to the limiting diffusive term. We leave this possibility to future research directions.

\section{Appendix.}

8.1. Stability analysis of first order IMEX schemes. For the subsequent analysis we restrict to the linear case $p(u)=u$ and $q(u)=0$

$$
\begin{aligned}
& u_{t}=-\left(v+\mu u_{x}\right)_{x}+\mu u_{x x}, \\
& \varepsilon^{2} v_{t}=-u_{x}-v
\end{aligned}
$$

We now look for a Fourier solution of the form $u=\hat{u}(t) \exp (i \xi x), v=\hat{v}(t) \exp (i \xi x)$ and inserting the ansatz into systems (1.1), the evolution equations are

$$
\begin{aligned}
\hat{u}_{t} & =-i \xi \hat{v}+\xi^{2} \mu \hat{u}-\xi^{2} \mu \hat{u} \\
\varepsilon^{2} \hat{v}_{t} & =-i \xi \hat{u}-\hat{v}
\end{aligned}
$$

It is convenient to rewrite the system using the variable $\hat{w}=-i \hat{v} / \xi$ in place of $\hat{v}$ so the system becomes

$$
\begin{aligned}
\hat{u}_{t} & =\xi^{2}(\hat{w}+\mu \hat{u})-\xi^{2} \mu \hat{u}, \\
\varepsilon^{2} \hat{w}_{t} & =-\hat{u}-\hat{w} .
\end{aligned}
$$

We apply the first order IMEX method based on explicit and implicit Euler schemes to get

$$
\begin{aligned}
\hat{u}^{n+1} & =\hat{u}^{n}+\Delta t \xi^{2}\left(\hat{w}^{n}+\mu \hat{u}^{n}\right)-\Delta t \xi^{2} \mu \hat{u}^{n+1}, \\
\varepsilon^{2} \hat{w}^{n+1} & =\varepsilon^{2} \hat{w}^{n}-\Delta t \hat{u}^{n+1}-\Delta t \hat{w}^{n+1}, \\
21 &
\end{aligned}
$$


which after manipulation can be written explicitly in the form

$$
\begin{aligned}
\hat{u}^{n+1} & =\hat{u}^{n}+\frac{\Delta t \xi^{2}}{1+\Delta t \xi^{2} \mu} \hat{w}^{n}, \\
\hat{w}^{n+1} & =\frac{\varepsilon^{2}\left(1+\Delta t \xi^{2} \mu\right)-\Delta t^{2} \xi^{2}}{\left(\varepsilon^{2}+\Delta t\right)\left(1+\Delta t \xi^{2} \mu\right)} \hat{w}^{n}-\frac{\Delta t}{\varepsilon^{2}+\Delta t} \hat{u}^{n} .
\end{aligned}
$$

In order to study the stability of the method we compute the eigenvalues of the stability matrix

$$
R=\left(\begin{array}{cc}
1 & \frac{\Delta t \xi^{2}}{1+\Delta t \xi^{2} \mu} \\
-\frac{\Delta t}{\varepsilon^{2}+\Delta t} & \frac{\varepsilon^{2}\left(1+\Delta t \xi^{2} \mu\right)-\Delta t^{2} \xi^{2}}{\left(\varepsilon^{2}+\Delta t\right)\left(1+\Delta t \xi^{2} \mu\right)}
\end{array}\right) .
$$

We obtain the expressions

$$
\lambda_{ \pm}=\frac{1}{2}\left\{1+\alpha(1+\beta)-\beta \pm \sqrt{[1+\alpha(1+\beta)-\beta]^{2}-4 \alpha}\right\}
$$

with

$$
\alpha=\frac{\varepsilon^{2}}{\varepsilon^{2}+\Delta t}, \quad \beta=\frac{\Delta t \xi^{2}}{1+\mu \Delta t \xi^{2}} .
$$

It can be shown that $\left|\lambda_{ \pm}\right|<1$ when

$$
\alpha<\frac{1-2 \beta+\beta^{2}}{1+2 \beta+\beta^{2}} .
$$

The above inequality involves a third order polynomial in $\Delta t$

$$
-\xi^{4}(\mu-1)^{2} \Delta t^{3}+2 \xi^{2}\left(2 \varepsilon^{2} \xi^{2} \mu+1-\mu\right) \Delta t^{2}+\left(4 \varepsilon^{2} \xi^{2}-1\right) \Delta t<0 .
$$

The roots of this polynomial are given by $T_{0}=0$ and

$$
T_{ \pm}=\frac{1}{(\mu-1)^{2} \xi^{2}}\left\{2 \varepsilon^{2} \xi^{2} \mu-\mu+1 \pm 2 \varepsilon \xi \sqrt{\varepsilon^{2} \xi^{2} \mu^{2}-\mu+1}\right\} .
$$

Condition (8.8) can be satisfied only if the last two roots are positive. This is guaranteed when $2 \varepsilon|\xi|<1$ and so we have the time step restriction $\Delta t<T_{-}$. The largest stability region is obtained when $\mu=1$ for which we get

$$
\xi^{2} \Delta t<\frac{1}{4} \frac{\left(1-4 \xi^{2} \varepsilon^{2}\right)}{\varepsilon^{2} \xi^{2}}
$$

This relation is interpreted as follows. For a fixed $\varepsilon|\xi|<1 / 2$, the restriction on the time step is of parabolic type, since $|\xi| \sim 1 / \Delta x$ is the maximum Fourier mode represented on a grid of spacing $\Delta x$. The restriction on $\Delta t / \Delta x^{2}$ is less and less severe as $\varepsilon \rightarrow 0$.

The implicit treatment of the second equation stabilizes the explicit treatment of the first one, provided $\varepsilon$ is sufficiently small.

8.2. Analysis of second order stiffly accurate schemes. We have the following result:

TheOrem 8.1. Consider an IMEX Runge-Kutta scheme of type A. Then there exist no second-order tree stage scheme satisfying the conditions $b^{T} A^{-1}=e_{s}^{T}$ and $\tilde{b}^{T}=e_{s}^{T} \tilde{A}$.

Proof. We consider the classical second order conditions

$$
\begin{gathered}
\tilde{b}^{T} e=1, \quad b^{T} e=1, \\
\tilde{b}^{T} \tilde{c}=1 / 2, \quad b^{T} c=1 / 2, \\
\tilde{b}^{T} c=1 / 2, \quad b^{T} \tilde{c}=1 / 2,
\end{gathered}
$$


with $c=A \mathbf{1}$ and $\tilde{c}=\tilde{A} \mathbf{1}$ and the conditions $b^{T} A^{-1}=e_{s}^{T}$ and $\tilde{b}^{T}=e_{s}^{T} \tilde{A}$.

For $s=3$ the Butcher tableau of a stiffly accurate IMEX R-K of type A is

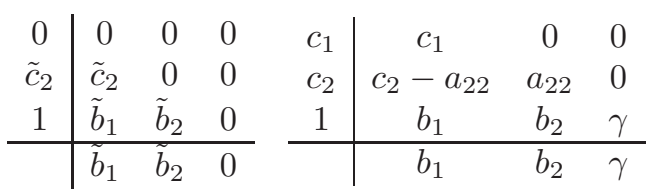

(note that stiff accuracy implies $c_{s}=\tilde{c}_{s}=1$ ) and from (8.10) the resulting system of equations can be explicitly written

$$
\begin{array}{cc}
\tilde{b}_{1}=1-\tilde{b}_{2}, & b_{1}=1-\gamma-b_{2}, \\
\tilde{b}_{2} \tilde{c}_{2}=1 / 2, & \left(1-\gamma-b_{2}\right) c_{1}+b_{2} c_{2}=1 / 2-\gamma, \\
\left(1-\tilde{b}_{2}\right) c_{1}+\tilde{b}_{2} c_{2}=1 / 2, & b_{2} \tilde{c}_{2}=1 / 2-\gamma .
\end{array}
$$

In order to solve system (8.11) we can compute the coefficients as follows

$$
\tilde{b}_{2}=1 /\left(2 \tilde{c}_{2}\right), \quad b_{2}=(1-2 \gamma) /\left(2 \tilde{c}_{2}\right),
$$

and

$$
\begin{gathered}
b_{2}\left(c_{2}-c_{1}\right)=1 / 2-\gamma-c_{1}+c_{1} \gamma, \\
\tilde{b}_{2}\left(c_{2}-c_{1}\right)=1 / 2-c_{1} .
\end{gathered}
$$

Substituting $\tilde{b}_{2}$ and $b_{2}$ in (8.12) we get

$$
\begin{aligned}
& \frac{\left(c_{2}-c_{1}\right)}{2 \tilde{c}_{2}}=\frac{1 / 2-\gamma-c_{1}+c_{1} \gamma}{1-2 \gamma}, \\
& \frac{\left(c_{2}-c_{1}\right)}{2 \tilde{c}_{2}}=1 / 2-c_{1} .
\end{aligned}
$$

Now, comparing and equating the two expressions we have $\gamma=0$ and it is impossible because the matrix

\begin{tabular}{|c|c|c|c|c|c|c|c|}
\hline 0 & 0 & 0 & 0 & $1 / 4$ & $1 / 4$ & 0 & 0 \\
\hline $1 / 2$ & $1 / 2$ & 0 & 0 & $1 / 4$ & 0 & $1 / 4$ & 0 \\
\hline 1 & $1 / 2$ & $1 / 2$ & 0 & 1 & $1 / 3$ & $1 / 3$ & $1 / 3$ \\
\hline & $1 / 3$ & $1 / 3$ & $1 / 3$ & & $1 / 3$ & $1 / 3$ & $1 / 3$ \\
\hline
\end{tabular}
$A$ is invertible.

\subsection{Second and third order IMEX schemes.}

1. Second order IMEX schemes:

- $\operatorname{ARS}(2,2,2)$ scheme, 1 ]

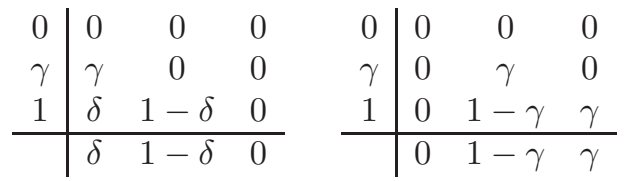

with $\gamma=(2-\sqrt{2}) / 2$ and $\delta=1-1 /(2 \gamma)$.

- SSP2-(3,3,2) scheme, 36.

2. Third order IMEX schemes:

- $\operatorname{ARS}(4,4,3)$ scheme, 1 .

\begin{tabular}{c|cccccc|ccccc}
0 & 0 & 0 & 0 & 0 & 0 & 0 & 0 & 0 & 0 & 0 & 0 \\
$1 / 2$ & $1 / 2$ & 0 & 0 & 0 & 0 & $1 / 2$ & 0 & $1 / 2$ & 0 & 0 & \\
$2 / 3$ & $11 / 18$ & $1 / 18$ & 0 & 0 & 0 & $2 / 3$ & 0 & $1 / 6$ & $1 / 2$ & 0 & 0 \\
$1 / 2$ & $5 / 6$ & $-5 / 6$ & $1 / 2$ & 0 & 0 & $1 / 2$ & 0 & $-1 / 2$ & $1 / 2$ & $1 / 2$ & 0 \\
1 & $1 / 4$ & $7 / 4$ & $3 / 4$ & $-7 / 4$ & 0 & 1 & 0 & $3 / 2$ & $-3 / 2$ & $1 / 2$ & $1 / 2$ \\
\hline & $1 / 4$ & $7 / 4$ & $3 / 4$ & $-7 / 4$ & 0 & & 0 & $3 / 2$ & $-3 / 2$ & $1 / 2$ & $1 / 2$
\end{tabular}


- $\operatorname{BPR}(3,5,3)$ scheme

\begin{tabular}{|c|c|c|c|c|c|c|c|c|c|c|c|}
\hline 0 & 0 & 0 & 0 & 0 & 0 & 0 & 0 & 0 & 0 & 0 & 0 \\
\hline 1 & 1 & 0 & 0 & 0 & 0 & 1 & $1 / 2$ & $1 / 2$ & 0 & 0 & \\
\hline $2 / 3$ & $4 / 9$ & $2 / 9$ & 0 & 0 & 0 & $2 / 3$ & $5 / 18$ & $-1 / 9$ & $1 / 2$ & 0 & O \\
\hline 1 & $1 / 4$ & 0 & $3 / 4$ & 0 & 0 & 1 & $1 / 2$ & 0 & 0 & $1 / 2$ & 0 \\
\hline 1 & $1 / 4$ & 0 & $3 / 4$ & 0 & 0 & 1 & $1 / 4$ & 0 & $3 / 4$ & $-1 / 2$ & $1 / 2$ \\
\hline & $1 / 4$ & 0 & $3 / 4$ & 0 & , & & $1 / 4$ & 0 & $3 / 4$ & $-1 / 2$ & $1 / 2$ \\
\hline
\end{tabular}

\section{REFERENCES}

[1] U. Ascher, S. Ruuth, R. J. Spitheri, Implicit-explicit Runge-Kutta methods for time dependent Partial Differential Equations. Appl. Numer. Math. 25, (1997), pp. 151-167.

[2] C. Bardos, R. Santos, R. Sentis, Diffusion approximation and computation of the critical size of a transport operator, Trans. Amer. Math. Soc., 284 (1984), 617649.

[3] M. Bennoune, M. Lemou, L. Mieussens, Uniformly stable numerical schemes for the Boltzmann equation preserving the compressible Navier-Stokes asymptotics, J. Comp. Phys., 227 (2008), 3781-3803.

[4] A. Bensoussan, J.-L. Lions, G. Papanicolaou, Boundary layers and homogenization of transport processes, J. Pub. Res. Inst. Math. Sci Kyoto, 15 (1979), 53157.

[5] S. Boscarino, Error Analysis of IMEX Runge-Kutta Methods Derided from Differential Algebraic Systems, SIAM J. Numer. Anal. Vol. 45, No 4, pp. 1600-1621 (2007).

[6] S. Boscarino, On an accurate third order implicit-explicit Runge-Kutta method for stiff problems, Appl. Num. Math. 59 (2009) 15151528

[7] S. Boscarino, L. Pareschi, G. Russo, IMEX Runge-Kutta schemes and hyperbolic systems of conservation laws with stiff diffusive relaxation, ICNAAM, AIP Conference Proceedings 1168, (2009) 1106-1111.

[8] S. Boscarino, G. Russo, On a class of uniformly accurate IMEX Runge-Kutta schemes and applications to hyperbolic systems with relaxation, SIAM J. Sci. Comput., 31, 3, pp. 1926-1945, (2009).

[9] C. Buet, S. Cordier, An asymptotic preserving scheme for hydrodynamics radiative transfer models, Numerische Math. 108, 2, pp.199-221, (2007).

[10] J.A. Carrillo, T. Goudon, P. Lafitte, F. Vecil. Numerical schemes of diffusion asymptotics and moment closures for kinetic equations. J. Sci. Comput., 36, pp.113-149, (2008).

[11] M.H. Carpenter, C.A. Kennedy, Additive Runge-Kutta schemes for convection-diffusion-reaction equations Appl. Numer. Math. 44 (2003), no. 1-2, 139-181.

[12] K.M. Case, P.F. Zweifel, Linear Transport Theory. Addison-Wesley, Reading, MA (1997)

[13] F. Cavalli, G. Naldi, G. Puppo, M. Semplice, High order relaxation schemes for non linear diffusion problems. SIAM Journal on Numerical Analysis, pp. 2098-2119, 2007, Vol. 45, N. 5, ISSN: 0036-1429

[14] S. Chandrasekhar, Radiative transport. Dover, New York (1960)

[15] G. Q. Chen, D. Levermore, T. P. Liu, Hyperbolic conservations laws with stiff relaxation therms and entropy. Comm. Pure Appl. Math., 47, (1994), pp. 787-830.

[16] P. Degond, S. Jin, A smooth transition model between kinetic and diffusion equations. SIAM J. Numer. Anal., 42, 6, pp.2671-2687, (2005).

[17] P. Degond, Asymptotic preserving schemes for fusion modeling CEMRACS'10 research achievements: Numerical modeling of fusion, Volume 32, October 2011.

[18] Dormand, J. R.; Prince, P. J. (1980), A family of embedded Runge-Kutta formulae, Journal of Computational and Applied Mathematics 6 (1): 1926,

[19] L. Gosse, G. Toscani, Asymptotic-preserving and well-balanced schemes for radiative transfer and the Rosseland approximation. Numer. Math., 98, 2, pp.223-250, (2004).

[20] J. L. Graveleau, P. Jamet, A finite difference approach to some degenerate nonlinear parabolic equations. SIAM J. Appl. Math., 20 (1971), pp. 199-223.

[21] E. Hairer, G. Wanner, Solving Ordinary Differential Equation II: stiff and Differential Algebraic Problems. Springer Series in Comput. Mathematics, Vol. 14, Springer-Verlag 1991, Second revised edition 1996.

[22] E. Hairer, S. P. Norsett, G. Wanner, Solving Ordinary Differential Equation I: Nonstiff Problems. Springer Series in Comput. Mathematics, Vol. 8, Springer-Verlag 1987, Second revised edition 1993.

[23] S. Jin, Asymptotic preserving (AP) schemes for multiscale kinetic and hyperbolic equations: a review. Lecture Notes for Summer School on "Methods and Models of Kinetic Theory", Porto Ercole (Grosseto, Italy), June 2010. Rivista di Matematica dell'Universita di Parma, to appear.

[24] S. Jin, F. Filbet, A class of asymptotic preserving schemes for kinetic equations and related problems with stiff sources, J. Comp. Phys. 229, 7625-7648, 2010.

[25] S. Jin, L. Pareschi, G. Toscani, Diffusive Relaxation Schemes for Multiscale Discrete-Velocity Kinetic Equations SIAM J on Numer. Anal., Vol. 35, No. 6 (Dec., 1998), pp. 2405-2439

[26] S. Jin, L. Pareschi, G. Toscani, Uniformly accurate diffusive relaxation schemes for transport equations. SIAM J. Numer. Anal., 38, 3, pp.913-936, (2000).

[27] S. Jin, L. Pareschi, Discretization of the multiscale semiconductor Boltzmann equation by diffusive relaxation schemes. J. Comp. Phys., 161, pp.312-330, (2000).

[28] A. Klar, An asymptotic-induced scheme for non stationary transport equations in the diffusive limit. SIAM J. Numer. Anal. 35, no. 3, (1998), pp. 1073-1094. 
[29] A. Klar, A numerical method for kinetic semiconductor equations in the drift-diffusion limit. SIAM Journal on Scientific Computing, 20, 5, pp.1696-1712, (1998).

[30] A. Klar, An asymptotic preserving numerical scheme for kinetic equations in the low mach number limit. Siam J Numer Anal, 36, 5, pp.1507-1527, (1999).

[31] P. Lafitte, G. Samaey, Asymptotic-preserving projective integration schemes for kinetic equations in the diffusion limit, preprint (2010).

[32] M. Lemou, L. Mieussens, A new asymptotic preserving scheme based on micro-macro formulation for linear kinetic equations in the diffusion limit. SIAM Journal on Scientific Computing, 2010, vol. 31, no. 1, pp. 334-368.

[33] T. P. Liu, Hyperbolic conservation laws with relaxation, Comm. MAth. Phys. 108, (1987), pp. 153-175.

[34] G. Naldi, L. Pareschi, Numerical Schemes for Hyperbolic Systems of Conservation Laws with Stiff Diffusive Relaxation SIAM J. on Numer. Anal., Vol. 37, No. 4 (2000), pp. 1246-1270

[35] G. Naldi, L. Pareschi, Numerical schemes for kinetic equations in diffusive regimes App. Math. Letters, Vol. 11, (1998), pp. 29-35

[36] L. Pareschi, G. Russo, Implicit-Explicit Runge-Kutta schemes and applications to hyperbolic systems with relaxations. Journal of Scientific Computing Volume: 25, Issue: 1, October, 2005, pp. 129-155

[37] L. Pareschi, G. Russo, Efficient asymptotic preserving deterministic methods for the Boltzmann equation, AVT-194 RTO AVT/VKI, Models and Computational Methods for Rarefied Flows, Lecture Series held at the von Karman Institute, Rhode St. Gense, Belgium, 24 -28 January (2011).

[38] W. Ruijgrok, T.T. Wu, A completely solvable model of the nonlinear Boltzmann equation., Phys. A, 113 (1982), pp. 401-416.

[39] L. Ryzhik, G. Papanicolaou, J.B. Keller Transport equations for elastic and other waves in random media Wave motion 24 (1996), pp. 327-370

[40] C-W. Shu, Essentially Non Oscillatory and Weighted Essentially Non Oscillatory schemes for hyperbolic conservation laws, in Advanced numerical approximation of nonlinear hyperbolic equations, Lecture Notes in Mathematics, 1697, (2000).

[41] A.N. Tikhonov, A.B. Vasl'eva, A.G. Sveshnikov, Differential Equations, Trans. from the Russian by A.B. Sossinskij, Springer Verlag, 1985, 238pp. 